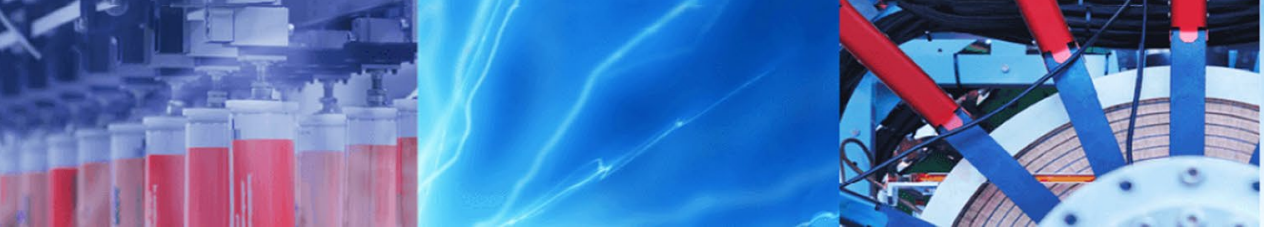

Research Article

\title{
Hopf bifurcation, offset boosting and remerging Feigenbaum trees in an autonomous chaotic system with exponential nonlinearity
}

\author{
K. Marcel Wouapi ${ }^{1} \cdot$ B. Hilaire Fotsin ${ }^{1} \cdot$ K. Florent Feudjio ${ }^{3} \cdot$ T. Zeric Njitacke ${ }^{1,2}$
}

Received: 11 August 2019 / Accepted: 15 November 2019 / Published online: 28 November 2019

(c) Springer Nature Switzerland AG 2019

\begin{abstract}
The present paper executes an analysis of a relatively simple chaotic system with exponential nonlinearity. This dimensionless dynamic system has been obtained from the chaotic circuit introduced by Ma and collaborators (nonlinear Dyn. https://doi.org/10.1007/s11071-018-4307-x) using scale transformation. The major dynamic properties of this model have been investigated, in particular, the equilibrium points and the dissipation property of the model explored. Based on usual diagnostic tools such as phase portraits, bifurcation diagrams, frequency spectrum, Lyapunov spectrum and basins of attraction, the nonlinear behaviours of the model have been highlighted. We demonstrated that the threedimensional autonomous system experiences the especial feature of subcritical Hopf bifurcation (when the control parameter exceeds a critical value); equally the period-doubling bifurcation, saddle-node bifurcation, offset boosting, reverse period doubling and antimonotonicity have been found. With the use of the normal form theory (Hassard algorithm), a formula for finding the direction of the Hopf bifurcation and the stability of bifurcating periodic solutions is presented. Furthermore, by using the Marsden-MacCracken index, we show that this Hopf bifurcation at equilibria is non-degenerate. At the end, the experimental results are given to further support the theoretical analysis.
\end{abstract}

Keywords Chaotic system with exponential nonlinearity · Route to chaos · Antimonotonicity · Offset boosting · Hopf bifurcation · Experimental study

\section{Introduction}

In several fields of studies including image processing [1], chemical reactions [2], telecommunication [3], biology $[4,5]$, just to name a few, chaos theory has many applications. As an example in biology, chaotic systems are used to produce electrical activities in the neuron by setting appropriate circuits, so that these neuronal circuits [6-9] can be constructed to examine the signal exchange and communication between neurons. From the innovative works of Lorentz [10] and Rössler [11], it was established that this striking and interesting phenomenon (chaos) could be found in systems of autonomous ordinary differential equations having a minimum of three variables with one or two nonlinearities. Over the years, many other autonomous/non-autonomous chaotic/hyperchaotic systems have been proposed and investigated [12-21]. Recently, it has been proven that, by just changing the initial conditions in a chaotic system, it could cause the switch from one attractor to another in the phase space. This phenomenon, better known as the coexistence of attractors, has been particularly investigated in nonlinear systems such as Jerk, Duffing, Lorentz, Rössler and many other systems. By carefully investigating this fascinating

$\triangle$ K. Marcel Wouapi, marcelwk2000@yahoo.fr | ${ }^{1}$ Unité de Recherche de Matière Condensée, d'Electronique et de Traitement du Signal (URMACETS), Department of Physics, University of Dschang, P.O. Box 67, Dschang, Cameroon. '2Unité de Recherche d'Automatique et Informatique Appliquée (LAIA), Department of Electrical Engineering, IUT-FV Bandjoun, University of Dschang, Dschang,

Cameroon. ${ }^{3}$ Laboratoire d'Energie et des Systemes Electriques et Electroniques, Department of Physics, University of Yaounde I, PO Box 812, Yaounde, Cameroon.

SN Applied Sciences (2019) 1:1715 | https://doi.org/10.1007/s42452-019-1702-y 
phenomenon (i.e. coexistence of attractors), researchers have shown the existence of bi-stability [18], multi-stability [4], extreme multi-stability [19] and, more recently, Sprott et al. [21] have proved the existence of "mega-stability", thus reflecting the simultaneous presence of two or more attractors (self-excited or hidden) for the same range of system parameters.

Recently, Ma et al. [22] proposed a 3D autonomous system with four constant parameters and one nonlinear term synthesized with a diode. As explained by [15, 23], this type of special nonlinearity is commonly used to simplify the implementation and to reduce the bulk and the cost of the circuit. Based on the exponential nonlinearity, this particular system shows potential to develop interesting behaviours including non-degenerate HB. It is important to recall that, the transition from stability to instability in some contests is explicitly linked to the disappearance or birth of a periodic orbit. When a change of this nature occurs in a system, it is said that there is the appearance of the Poincare-Andronov-Hopf bifurcation, better known as the Hopf bifurcation (HB). This bifurcation can provide a good explanation for many physical phenomena that are usually encountered because it characterizes the simplest mechanism of transition from a stationary regime to oscillations (or oscillations at a stationary regime). As an example in neurodynamics, the possible mechanisms for highlighting how a nervous system reacts instantly to excitation are described by sudden changes observed in the dynamic behaviour of this system. This change in the behaviour of the nervous system is due to the appearance of a phenomenon called Hopf bifurcation (HB) [24]. This is the major reason why the study and mastery of this type of bifurcation in particular is very important in the theory of bifurcations.

In several disciplines of science and engineering, the HB analysis of 3D systems (especially with quadratic or cubic nonlinearity) has received increasing attention of numerous researchers (such as Chen's system [25], biological system [26], Lü system [27, 28], Lui system [29], Kingni-Jafari system [30], and so on and so forth). Even if the Hassard algorithm dates before 1978, a very few theoretical analysis (by using the normal form theory) is found in the literature for the autonomous chaotic systems with exponential nonlinearity to the best of our knowledge. From these observations, the present work considers the dynamics of a 3D system with an exponential nonlinear term recently introduced by [22]. In the latter literature, the authors investigate the problem of crack synchronization (synchronization and desynchronization) between two chaotic circuits under field coupling. Hence, two chaotic circuits are connected through voltage coupling and magnetic field coupling was realized between two isolated inductance coils. The authors show that field coupling could inflect the synchronization behaviours of chaotic circuits. In order to clarify these results, mathematic proof, numeric and simulations (using Proteus 8 Professional-Schematic Capture tool) were presented. Nevertheless, these interesting results were restricted to the synchronization of the chaotic oscillators and made no mention of the chaotic mechanism. Also, to throw more light on the dynamics of this system, our aim here is threefold: (1) investigate the dynamics of the system of [22] and explain the chaotic mechanism; (2) limit the region in the parameter space in which the model exhibits complex dynamical behaviours (antimonotonicity, offset boosting and Hopf bifurcation); and (3) execute an experimental laboratory study of the autonomous system to confirm the theoretical analysis.

As a general motivation, this study at first contributes to enrich the literature with a new simple 3D autonomous system with exponential nonlinearity presenting the phenomenon of Hopf bifurcation (HB). Secondly, the model represents an interesting example for students studying nonlinear dynamics and chaos in particular, because of the extreme simplicity of its mathematical equations and the captivating phenomena it can display. Thirdly, we are developing the necessary tools for making practical circuits of this oscillator.

The rest of this work is organized as follows. Section 2 describes the mathematical model of the system under investigation and figure out the possible existence of attractors. The stability of the stationary points is analysed, and the possibility of Hopf bifurcation is obtained. In Sect. 3 by applying the normal form theory, the Marsden and MacCracken index, Hopf bifurcation is investigated. The direction of the Hopf bifurcation and stability of the bifurcating periodic solutions are analysed in detail. In Sect. 4, the bifurcation features of the system are investigated numerically. In Sect. 5, an appropriate analogue circuit is proposed for the examination of the dynamic behaviour of the system. The correspondences are established between the coefficients of the mathematical model and the analogue simulator components. Experimental and numerical are equivalent to yield a very good results. Finally, the conclusion is presented in Sect. 6 where we indicate possible future work.

\section{Description and analysis of the model}

\subsection{The model}

The mathematical expression of the simple chaotic system [22] considered in this work is shown by the following set of coupled differential equations: 


$$
\left\{\begin{array}{l}
\dot{x}=a x-y \\
\dot{y}=c(x-z) \\
\dot{z}=d\left[y-b\left(e^{z}-1\right)\right]
\end{array}\right.
$$

In system (1), $a, b, c$ and $d$ are positive tuneable parameters, while $x, y$ and $z$ are state variables which can be used to describe voltages across the capacitors or the currents induced through inductors. From the above, we can see that only the state variable $z$ appears in the exponential nonlinearity of the model. The complicated behaviours exhibited by the whole system are caused by the availability of this nonlinearity. Briefly recall that, the first Jerk systems were developed by Sprott [31, 32] and are parts of the category of 3D dynamic system called "elegant"; these systems are subject of several researches in different areas of science and even in engineering [31-36]. Jerk systems arouse such interest because their mathematical expressions are defined by a third-order differential equation describing the time evolution of a single scalar variable $u(\tau)$ such that: $\ddot{u}=J(u, \dot{u}, \ddot{u})$ where $J$ represents "jerk" or "jolt", the single scalar variable $u(\tau)$ represents the displacement, the first derivative $\dot{u}(\tau)$ is the velocity, and the second derivative of the displacement $\ddot{u}(\tau)$ represents the acceleration in a mechanical system. We can see that the jerk function is actually the derivative of the acceleration [31]. The careful dynamic study of such system can be very interesting because it can hide exceptional phenomena such as chaos and synchronization. Thus, several rich and very captivating phenomena such as crisis route to chaos, period doubling, periodic window, intermittency scenario, antimonotonicity and multiple coexisting attractors have been highlighted in jerk system by researchers like Sprott, Jafari, Akgul, Kengne, Njitacke [13] and many others. Considering this as motivation, it is important to show that the system (1) can be put in the general form of jerk systems in order to be able to find in the study of its dynamics important phenomena. So, by deriving twice the third equation of the system (1) we have $\dddot{z}=c d(\dot{x}-\dot{z})-b d \ddot{z} \mathrm{e}^{z}-b d \dot{z}^{2} \mathrm{e}^{z}$, knowing that:

$\dot{x}=\frac{a}{c d} \ddot{z}+\left(\frac{a b}{c} \mathrm{e}^{z}-\frac{1}{d}\right) \dot{z}+a z-b\left(\mathrm{e}^{z}-1\right) \quad$ and $\dot{y}=\frac{1}{d} \ddot{z}+b \dot{z} \mathrm{e}^{z}$,

weobtain $\ddot{z}=\left(a-b d \mathrm{e}^{z}\right) \ddot{z}+\left(a b d \mathrm{e}^{z}-c(1+d)-b d \dot{z} \mathrm{e}^{z}\right) \dot{z}+$ $a c d z-b c d\left(\mathrm{e}^{z}-1\right)$. Let us define $u=z, v=\dot{z}$ and $w=\ddot{z}$, system (1) becomes:

$\dddot{u}=a \ddot{u}-c(1+d) \dot{u}+a d c u+f(u, v, w) \quad$ where $f(u, v, w)=b c d+\left(a v-v^{2}-w-c\right) b d \mathrm{e}^{u}$

For the initial condition $(-0.1,0.2,1.1)$, this $3 \mathrm{D}$ system has a chaotic attractor for the particular parameters $a=0.5, b=0.001, c=1$ and $d=2$.
In the remaining part of this work, we will use the system (1), because the nonlinearity of the system (2) is much more complex and expensive to realize.

\subsection{Dissipation and presence of attractors}

Many studies have proven that nonlinear dissipative systems can be dissipative and thus support chaotic attractors [37-39]. In short, the volume contraction rate of a dynamical system can be studied by the divergence of its vector field; it is defined as:

$\Pi=\Delta V=\frac{\partial \dot{x}}{\partial x}+\frac{\partial \dot{y}}{\partial y}+\frac{\partial \dot{z}}{\partial z}=a-b d \mathrm{e}^{z}$

For $\Pi=0$, phase space volume is kept and the system is conservative, when $\Pi>0$ the volume in phase space expands. Where $\Pi<0$, local volumes in the phase space are contracted and the system has attracting sets. Verifying this condition for Eq. (1), we obtain $\frac{\mathrm{d} V}{\mathrm{~d} \tau}=\exp \left[\left(a-d b \mathrm{e}^{z}\right) \tau\right]$, that is $V(\tau)=V_{0} \exp \left[\left(a-d b \mathrm{e}^{z}\right) \tau\right]$, where $V_{0}=V(t=0)=\frac{1}{a-d b \mathrm{e}^{2}}$ is the initial value of the system volume. If the condition $a<d b \mathrm{e}^{z}$ is satisfied, then $\lim _{\tau \rightarrow+\infty}\left[\exp \left(\left(a-d b \mathrm{e}^{z}\right) \tau\right)\right]=0$, which means that all system trajectories will be limited to the point of zero volume set, and its behaviour will be fixed in the attractor when $\tau \rightarrow+\infty$. This is a necessary property for the existence of chaotic attractors.

\subsection{Stationary points}

With a view to analyse the system dynamics, a good start is to find its possible equilibria, and then to characterize the local dynamical behaviours of the system orbits near these points. As seen in Eq. (1), the equilibrium points can be approached by resolving the following equations:

$$
\left\{\begin{array}{l}
x=z \\
y=a x \\
z=b\left(e^{z}-1\right) .
\end{array}\right.
$$

That is, Eq. (4) is a transcendental nonlinear system, it is impossible to be analytically resolved. However, the non-trivial fixed points can be gotten by making recourse to numerical methods, for instance, by using the MAPLE built-in function, "solve" for certain systems parameters.
It is obvious that the origin $O(0,0,0)$ is the only trivial stationary point. Note that the non-trivial solution $S\left(x_{0}, y_{0}, z_{0}\right)$ is defined solely by the two parameters $a$ and $b$. With the 
particular values of parameters $a=0.2$ and $b=0.001$, the non-trivial solution is $S(7.2848,1.4569,7.2848)$. We linearize the system (1) around this equilibrium $\left(x_{0}, y_{0}, z_{0}\right)$, and the following $3 \times 3$ Jacobian matrix is obtained:

$J=\left(\begin{array}{ccc}a & -1 & 0 \\ c & 0 & -c \\ 0 & d & -d b \mathrm{e}^{x_{0}}\end{array}\right)$,

and thus the characteristic equation $(\operatorname{det}(J-\lambda l)=0)$ can be easily calculated as follows:

$\lambda^{3}-\left(a-d b \mathrm{e}^{x_{0}}\right) \lambda^{2}-\left(d b a \mathrm{e}^{x_{0}}-d c-c\right) \lambda+d c\left(b \mathrm{e}^{x_{0}}-a\right)=0$.

Table 1 shows the eigenvalues (roots of Eq. 6) obtained with the help of the Newton-Raphson method for modifying the parameter $c$ in the interval $0.05 \leq c \leq 1.6$, the order parameters keeping $a=0.2, b=0.001$ and $d=0.5$. This table explicitly shows that the real part of the eigenvalues always has positive signs for the origin $O(0,0,0)$; consequently, it is unstable. We observe that for values of $c \geq 0.6$, the fixed point $S$ has eigenvalues all with a negative real part; therefore, it is a stable focus. Eigenvalues with different real parts are gotten for values of $c \leq 0.41$; in this case, $S$ is considered as unstable focus. Figure 1 provides the representation of the eigenvalues solutions of Eq. (6) in the complex plane (Real $(\lambda)$, Imag $(\lambda))$ work out for $0.2 \leq c \leq 1$ while conserving the values defined previously. It is observed in this figure that the locus meets the imaginary axis and thus proposes chances of the Hopf bifurcation.

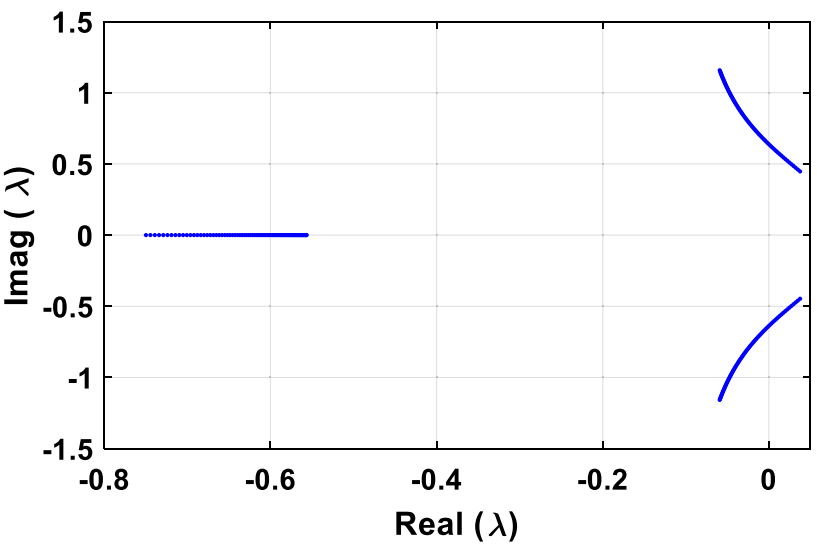

Fig. 1 Representation of the eigenvalues solutions of Eq. (6) in the complex plane $(\operatorname{Real}(\lambda)$, Imag $(\lambda))$ for $0.2 \leq c \leq 1$, while keeping $a=0.2, b=0.001, d=0.5\left(x_{0}=7.28479\right)$. Provided that $J$ is a real matrix, complex eigenvalues occur in complex conjugate pairs responsible of the symmetry observed along the real axis. The locus intersects the imaginary axis and thus suggests the possibility of Hopf bifurcation

\section{Hopf bifurcation (HB) analysis}

The priority of this section is to study the occurrence of a pair of the complex conjugate feature of an equilibrium state across the imaginary axis. Thus, we perform the analysis of parametric variations with reference to dynamical bifurcations at the equilibrium $S\left(x_{0}, y_{0}, z_{0}\right)$ by employing the classical form theory and symbolic computations [40, 41]. Considering Fig. 1 here, we will use the system (1) with the below parameter values: $a=0.2, b=0.001, d=0.5$ and $x_{0}=7.28479$.

Table 1 Fixed points, eigenvalues and stability computed for some discrete values of the bifurcation control parameter $c$

\begin{tabular}{|c|c|c|c|}
\hline $\begin{array}{l}\text { Values of the bifurcation param- } \\
\text { eter }(c)\end{array}$ & $\begin{array}{l}\text { Non-trivial fixed points } \\
(S)\end{array}$ & $\begin{array}{l}\text { Eigenvalues at non-trivial fixed point } \\
\text { (S) } \lambda_{1}, \lambda_{2}, \lambda_{3}\end{array}$ & $\begin{array}{l}\text { Eigenvalues at the origin } \\
O(0,0,0) \lambda_{1}, \lambda_{2}, \lambda_{3}\end{array}$ \\
\hline$c=0.05$ & $(7.2848,1.457,7.2848)$ & $\begin{array}{l}-0.6957 \\
0.0833 \pm 0.1956 i \text { (unstable) }\end{array}$ & $\begin{array}{l}0.0759 \\
0.0618 \pm 0.2483 i \text { (unstable) }\end{array}$ \\
\hline$c=0.12$ & $(7.2848,1.457,7.2848)$ & $\begin{array}{l}-0.6534 \\
0.0622 \pm 0.3341 i \text { (unstable) }\end{array}$ & $\begin{array}{l}0.0699 \\
0.0648 \pm 0.4082 i \text { (unstable) }\end{array}$ \\
\hline$. c=0.26$. & $(7.2848,1.457,7.2848)$ & $\begin{array}{l}-0.5873 \\
0.0292 \pm 0.5268 i \text { (unstable) }\end{array}$ & $\begin{array}{l}0.0679 \\
0.0658 \pm 0.6137 i \text { (unstable) }\end{array}$ \\
\hline$c=0.41$ & $(7.2848,1.457,7.2848)$ & $\begin{array}{l}-0.5417 \\
0.0063 \pm 0.6899 i\end{array}$ & $\begin{array}{l}0.0673 \\
0.0661 \pm 0.7757 i \\
\text { (unstable) }\end{array}$ \\
\hline$c=0.6$ & $(7.2848,1.457,7.2848)$ & $\begin{array}{l}-0.5077 \\
-0.0107 \pm 0.8621 i(\text { stable })\end{array}$ & $\begin{array}{l}0.067 \\
0.0662 \pm 0.9416 i \\
\text { (unstable) }\end{array}$ \\
\hline$c=1$ & $(7.2848,1.457,7.2848)$ & $\begin{array}{l}-0.4736 \\
-0.0277 \pm 1.1521 i(\text { stable })\end{array}$ & $\begin{array}{l}0.0667 \\
0.0664 \pm 1.2192 i \\
\text { (unstable) }\end{array}$ \\
\hline$c=1.6$ & $(7.2848,1.457,7.2848)$ & $\begin{array}{l}-0.4533 \\
-0.0378 \pm 1.4895 i \\
\text { (stable) }\end{array}$ & $\begin{array}{l}0.0666 \\
0.0665 \pm 1.5449 i \text { (unstable) }\end{array}$ \\
\hline
\end{tabular}




\subsection{Existence of Hopf bifurcation}

Before giving our results, we state the following transversality condition to demonstrate that Hopf bifurcation occurs in a system $[42,43]$.

Suppose that the system $\dot{x}=f_{\mu}(x), x \in \mathbb{R}^{n}, \mu \in \mathbb{R}$ has an equilibrium $\left(x_{0}, \mu_{0}\right)$ at which the following properties are satisfied:

(1) $D_{x} f_{u_{0}}\left(x_{0}\right)$ has a simple pair of pure imaginary eigenvalues $\lambda(\mu)$ and $\bar{\lambda}(\mu)$, and other eigenvalues with negative real parts;

(2) $\left.\frac{\mathrm{d}}{\mathrm{d} \mu}(R(\lambda(u)))\right|_{\mu=\mu_{0}} \neq 0$.

Then, the system $\dot{x}=f_{\mu}(x), x \in \mathbb{R}^{n}, \mu \in \mathbb{R}$ has a Hopf bifurcation at the equilibrium $\left(x_{0}, \mu_{0}\right)$.

To begin our analysis, we move the equilibrium $S\left(x_{0}, y_{0}, z_{0}\right)$ of system (1) to the origin $O(0,0,0)$ under the following linear transformation.

$\left\{\begin{array}{l}x_{1}=x-x_{0} \\ y_{1}=y-y_{0} \\ z_{1}=z-z_{0}\end{array}\right.$

which changes system (1) into the following

$$
\left\{\begin{array}{l}
\dot{x}_{1}=a x_{1}-y_{1} \\
\dot{y}_{1}=c\left(x_{1}-z_{1}\right) \\
\dot{z}_{1}=d\left[y_{1}-b\left(\mathrm{e}^{x_{0}} \mathrm{e}^{z_{1}}-1\right)\right]+a d x_{0}
\end{array}\right.
$$

Its Jacobian matrix $M_{\mathrm{J}}$ at the equilibrium $O(0,0,0)$ is

$M_{J}=\left(\begin{array}{ccc}a & -1 & 0 \\ c & 0 & -c \\ 0 & d & -\gamma\end{array}\right)$

where $\gamma=b d \mathrm{e}^{x_{0}}$ (From Eq. (4), we can also deduce that $\gamma=d\left(a x_{0}+b\right)$.)

The characteristic equation corresponding to this Jacobian matrix is

$f(\lambda)=\lambda^{3}+(\gamma-a) \lambda^{2}+(c+c d-a \gamma) \lambda-a d c+\gamma c=0$

According to Fig. 1, there are two complex conjugate zeros of $f(\lambda)$ for $0.2 \leq c \leq 1$ (i.e. Equation (11) has a pair of purely imaginary conjugate roots $\lambda_{1,2}= \pm \mathrm{i} \omega_{0}$ ) (see Fig. 1). Let these two zeros be $\lambda_{1}=\omega_{0}$ i and $\lambda_{2}=-\omega_{0}$ i for positive real number $\omega_{0}$. Since the sum of the three zeros of $f(\lambda)$ is:

$\lambda_{1}+\lambda_{2}+\lambda_{3}=-(\gamma-a)$

We have $\lambda_{3}=a-\gamma$ (by considering the parameter values defined above, $\lambda_{3}<0$ ), which is on the margin of stability, where $\lambda_{1,2}= \pm i \omega_{0}$. On this margin, we substitute $\lambda_{1}=\omega_{0} i$ into the characteristic Eq. (11) and obtain: $(a-\gamma) \omega_{0}^{2}+c_{r}(\gamma-a d)+i\left(\left(c_{r}-a \gamma+c_{r} d\right) \omega_{0}-\omega_{0}^{3}\right)=0$

From the above affirmation, we can end by saying that such bifurcation can occur in the system when the following equation is verified:

$\omega_{0}=\sqrt{\frac{a \gamma(\gamma-a d)}{d \gamma-a}}=\sqrt{\frac{a d^{2}\left(a x_{0}+b\right)\left(a x_{0}+b-a\right)}{d^{2}\left(a x_{0}+b\right)-a}}$

Referring to the parameter values in Fig. 1, $\frac{a d^{2}\left(a x_{0}+b\right)\left(a x_{0}+b-a\right)}{d^{2}\left(a x_{0}+b\right)-a} \geq 0$.

We obtain the following critical value

$c_{r}=\frac{a \gamma(\gamma-a)}{d \gamma-a}=\frac{a d\left(a x_{0}+b\right)\left(d\left(a x_{0}+b\right)-a\right)}{d^{2}\left(a x_{0}+b\right)-a}$

Thus, the first condition for a HB in the sense of [24-30] is satisfied.

With reference to Eq. (10), one has

$$
\begin{gathered}
\lambda^{\prime}\left(c_{r}\right)=\frac{(a d-\gamma)\left((1+d) c_{r}-a \gamma-3 \omega_{0}^{2}\right)-2 \omega_{0}^{2}(1+d)(\gamma-a)}{\left(-a \gamma+c_{r}(d+1)-3 \omega_{0}^{2}\right)^{2}+4 \omega_{0}^{2}(\gamma-a)^{2}} \\
-\frac{I\left[(2(a d-\gamma)) \omega_{0}(\gamma-a)+\omega_{0}(1+d)\left((1+d) c_{r}-a \gamma-3 \omega_{0}^{2}\right)\right]}{\left(-a \gamma+c_{r}(d+1)-3 \omega_{0}^{2}\right)^{2}+4 \omega_{0}^{2}(\gamma-a)^{2}} .
\end{gathered}
$$

Therefore,

$R_{e}\left(\lambda^{\prime}\left(c_{r}\right)\right)=\frac{(a d-\gamma)\left((1+d) c_{r}-a \gamma-3 \omega_{0}^{2}\right)-2 \omega_{0}^{2}(1+d)(\gamma-a)}{\left(-a \gamma+c_{r}(d+1)-3 \omega_{0}^{2}\right)^{2}+4 \omega_{0}^{2}(\gamma-a)^{2}}$.

By considering the parameters fixed in the text (see Fig. 1), $R_{e}\left(\lambda^{\prime}\left(c_{r}\right)\right) \neq 0$.

Thus, the second status for a HB is join and the HB theorem withstand. Hence, when $c=c_{r}$, system (4) undergoes a $\mathrm{HB}$ at the equilibrium $\mathrm{O}(0,0,0)$, namely system (1) undergoes a $\mathrm{HB}$ at the equilibrium $\mathrm{S}$. The above affirmation is seen as such:

Theorem 1 (Existence of Hopf bifurcation). When the parameter $c$ witnesses the critical value $c_{r}$, system (4) undergoes a Hopf bifurcation at the equilibrium $O(0,0,0)$.

\subsection{Direction and stability}

After having proved the existence of the Hopf bifurcation in the system (1) for a particular range of the parameter, it is essential to be interested in the instability related to this type of bifurcation. Indeed, thanks to Fig. 1, we can deduce some observations concerning the stability of fixed points and bifurcations likely to appear in the model submitted to our study. It can easily be deduced that the Jacobian matrix possesses absolutely real coefficients; therefore, the symmetry observed along the real axis is the result of the appearance of complex conjugate pairs of eigenvalue. 
Looking at the evolution of the eigenvalues of the Jacobian matrix of the system, we note that there is a type of instability which describes well the phenomenon of the $\mathrm{HB}$ : intersection place with the imaginary axis where two conjugated complexes eigenvalues cross the imaginary axis simultaneously. We also note that real (non-complex) solutions are always negative.

Now, we will apply the method proposed by [40-43] to determine the direction and the stability of the HB.

The eigenvectors $v_{1}$ and $v_{3}$ associated, respectively, with $\lambda_{1}=i \omega_{0}$ and $\lambda_{3}=a-\gamma$ are

$v_{1}=\left[\begin{array}{l}1 \\ a-i \omega_{0} \\ \frac{c-\omega_{0}^{2}-i a \omega_{0}}{c}\end{array}\right] ; v_{3}=\left[\begin{array}{r}-\frac{1}{\gamma} \\ 1 \\ \frac{d}{a}\end{array}\right]$

Thus, the matrix $P=\left(\operatorname{Rev} v_{1},-\operatorname{Im} v_{1}, v_{3}\right)=\left(P_{i j}\right)_{1 \leq i, j \leq 3}$ is determined as follows:

$P=\left[\begin{array}{ccc}1 & 0 & -\frac{1}{\gamma} \\ a & \omega_{0} & 1 \\ \frac{c-\omega_{0}^{2}}{c} & \frac{a \omega_{0}}{c} & \frac{d}{a}\end{array}\right]$
$P_{1}\left(x_{2}, y_{2}, z_{2}\right)=M\left(A_{1} x_{2}+B_{1} y_{2}+C_{1} z_{2}+\omega_{0} D\right)$
$P_{2}\left(x_{2}, y_{2}, z_{2}\right)=M\left(A_{2} x_{2}+B_{2} y_{2}+C_{2} z_{2}-(\gamma+c) D\right)$
$P_{3}\left(x_{2}, y_{2}, z_{2}\right)=M\left(A_{3} x_{2}+B_{3} y_{2}+C_{3} z_{2}+\frac{\omega_{0} D}{\gamma}\right)$

in which $D=\left(1-\exp \left(\frac{\left(-\omega_{0}^{2}+c\right) x_{2}}{c}+\frac{a \omega_{0} y_{2}}{c}+\frac{d z_{2}}{a}\right)\right)$ and the parameters $A_{1}, B_{1}, C_{1}, A_{2}, B_{2}, C_{2}, A_{3}, B_{3}, C_{3}, M$ are described in the "Appendix" Eqs.(25-34).

System (15) can be rewritten as:

$\left\{\begin{array}{l}\dot{x}_{2}=-\omega_{0} y_{2}+F_{1}\left(x_{2}, y_{2}, z_{2}\right) \\ \dot{y}_{2}=\omega_{0} x_{2}+F_{2}\left(x_{2}, y_{2}, z_{2}\right) \\ \dot{z}_{2}=(a-\gamma) z_{2}+F_{3}\left(x_{2}, y_{2}, z_{2}\right)\end{array}\right.$

where

$$
\begin{aligned}
& F_{1}\left(x_{2}, y_{2}, z_{2}\right)=P_{1}\left(x_{2}, y_{2}, z_{2}\right)+\omega_{0} y_{2} \\
& F_{2}\left(x_{2}, y_{2}, z_{2}\right)=P_{2}\left(x_{2}, y_{2}, z_{2}\right)-\omega_{0} x_{2} \\
& F_{3}\left(x_{2}, y_{2}, z_{2}\right)=P_{3}\left(x_{2}, y_{2}, z_{2}\right)-(a-\gamma) z_{2}
\end{aligned}
$$

We note that:

and

$\frac{\partial^{i} F_{l}}{\partial \zeta^{i}}=\frac{\partial^{i} P_{l}}{\partial \zeta^{i}} \quad$ for $\quad i=2,3 \ldots ; l=1,2,3 ; \zeta=x_{2}, y_{2}, z_{2}$

and

$\frac{\partial^{i+j} F_{l}}{\partial \zeta^{i} \eta^{j}}=\frac{\partial^{i+j} P_{l}}{\partial \zeta^{i} \eta^{j}} \quad$ for $\quad i=1,2,3 \ldots ; j=1,2,3 \ldots ; l=1,2,3 ; \zeta, \eta=x_{2}, y_{2}, z_{2}$.

$\left[\begin{array}{l}x_{1} \\ y_{1} \\ z_{1}\end{array}\right]=P\left[\begin{array}{l}x_{2} \\ y_{2} \\ z_{2}\end{array}\right]$

Then, we obtain

$\left\{\begin{array}{l}\dot{x}_{2}=P_{1}\left(x_{2}, y_{2}, z_{2}\right) \\ \dot{y}_{2}=P_{2}\left(x_{2}, y_{2}, z_{2}\right) \\ \dot{z}_{2}=P_{3}\left(x_{2}, y_{2}, z_{2}\right)\end{array}\right.$
Then, we follow the same way put in place by Hassard et al. [42] to calculate the necessary quantities.

We can therefore get:

where 


$$
\begin{aligned}
g_{11}= & \frac{1}{4}\left[F_{20}^{1}+F_{02}^{1}+i\left(F_{20}^{2}+F_{02}^{2}\right)\right] \\
= & \frac{\sigma}{4 c^{2}}\left[-\omega_{0}\left(c-\omega_{0}^{2}\right)^{2}-a^{2} \omega_{0}^{3}+i\left((\gamma+c)\left(\left(c-\omega_{0}^{2}\right)^{2}+a^{2} \omega_{0}^{2}\right)\right)\right] \\
g_{02}= & \frac{1}{4}\left[F_{20}^{1}-F_{02}^{1}-2 F_{11}^{2}+i\left(F_{20}^{2}-F_{02}^{2}+2 F_{11}^{1}\right)\right] \\
= & \frac{\sigma}{4 c^{2}}\left[-\omega_{0}\left(c-\omega_{0}^{2}\right)^{2}+a^{2} \omega_{0}^{3}-2 a \omega_{0}(\gamma+c)\left(c-\omega_{0}^{2}\right)\right. \\
& \left.+i\left((\gamma+c)\left(c-\omega_{0}^{2}\right)^{2}-a^{2} \omega_{0}^{2}(\gamma+c)-2 a \omega_{0}^{2}\left(c-\omega_{0}^{2}\right)\right)\right] \\
g_{20}= & \frac{1}{4}\left[F_{20}^{1}-F_{02}^{1}+2 F_{11}^{2}+i\left(F_{20}^{2}-F_{02}^{2}-2 F_{11}^{1}\right)\right] \\
= & \frac{\sigma}{4 c^{2}}\left[-\omega_{0}\left(c-\omega_{0}^{2}\right)^{2}+a^{2} \omega_{0}^{3}+2 a \omega_{0}(\gamma+c)\left(c-\omega_{0}^{2}\right)\right. \\
& \left.+i\left((\gamma+c)\left(c-\omega_{0}^{2}\right)^{2}-a^{2} \omega_{0}^{2}(\gamma+c)+2 a \omega_{0}^{2}\left(c-\omega_{0}^{2}\right)\right)\right] \\
G_{21}= & \frac{1}{8}\left[F_{30}^{1}+F_{12}^{1}+F_{21}^{2}+F_{03}^{2}+i\left(F_{30}^{2}+F_{12}^{2}-F_{21}^{1}-F_{03}^{1}\right)\right] \\
= & \frac{\sigma}{8 c^{3}}\left[-\omega_{0}\left(c-\omega_{0}^{2}\right)^{3}-a^{2} \omega_{0}^{3}\left(c-\omega_{0}^{2}\right)+a \omega_{0}(\gamma+c)\left(c-\omega_{0}^{2}\right)^{2}+a^{3} \omega_{0}^{3}(\gamma+c)\right. \\
& \left.+i\left(a^{3} \omega_{0}^{3}(\gamma+c)+a^{2} \omega_{0}^{2}(\gamma+c)\left(c-\omega_{0}^{2}\right)+a \omega_{0}^{2}\left(c-\omega_{0}^{2}\right)^{2}+a^{3} \omega_{0}^{4}\right)\right] \\
h_{11}= & \frac{1}{4}\left(F_{20}^{3}+F_{02}^{3}\right)=-\frac{\sigma}{4 c^{2}}\left[\omega_{0} \gamma\left(c-\omega_{0}^{2}\right)^{2}+\gamma a^{2} \omega_{0}^{3}\right] \\
h_{20}= & \frac{1}{4}\left(F_{20}^{3}-F_{02}^{3}-2 i F_{11}^{3}\right)=\frac{\sigma}{4 c^{2}}\left[\gamma a^{2} \omega_{0}^{3}-\gamma \omega_{0}\left(c-\omega_{0}^{2}\right)^{2}+i\left(2 \gamma a \omega_{0}^{2}\left(c-\omega_{0}^{2}\right)\right)\right]
\end{aligned}
$$

In witch $\sigma=M \exp \left(\frac{\left(c-\omega_{0}^{2}\right) x_{2}+a \omega_{0} y_{2}}{c}+\frac{d z_{2}}{a}\right)$

Thus, we can calculate the following quantities

$$
\begin{aligned}
w_{11}= & -\frac{\sigma \gamma \omega_{0}}{4 c^{2}(a-\gamma)}\left[\left(c-\omega_{0}^{2}\right)^{2}+a^{2} \omega_{0}^{2}\right] \\
w_{20}= & \frac{\sigma \gamma \omega_{0}}{4 c^{2}\left(4 \omega_{0}^{2}+(a-\gamma)^{2}\right)}\left[(a-\gamma)\left(c-\omega_{0}^{2}\right)^{2}-a^{2} \omega_{0}^{2}+4 a \omega_{0}^{2}\left(c-\omega_{0}^{2}\right)\right. \\
& \left.+i\left(-2 a \omega_{0}(a-\gamma)\left(c-\omega_{0}^{2}\right)+2 \omega_{0}\left(c-\omega_{0}^{2}\right)^{2}-a^{2} \omega_{0}^{3}\right)\right] \\
G_{110}= & \frac{1}{2}\left[F_{10}^{1,1}+F_{01}^{2,1}+i\left(F_{10}^{2,1}-F_{01}^{1,1}\right)\right] \\
= & \frac{\sigma}{2 a c}\left[a d \omega_{0}(\gamma+c)-d \omega_{0}\left(c-\omega_{0}^{2}\right)+i\left(d(\gamma+c)\left(c-\omega_{0}^{2}\right)+a d \omega_{0}^{2}\right)\right] \\
G_{101}= & \frac{1}{2}\left[F_{10}^{1,1}-F_{01}^{2,1}+i\left(F_{10}^{2,1}+F_{01}^{1,1}\right)\right] \\
= & \frac{\sigma}{2 a c}\left[-a d \omega_{0}(\gamma+c)-d \omega_{0}\left(c-\omega_{0}^{2}\right)+i\left(d(\gamma+c)\left(c-\omega_{0}^{2}\right)-a d \omega_{0}^{2}\right)\right] \\
g_{21}= & G_{21}+\left(2 G_{110} w_{11}+G_{101} w_{20}\right)
\end{aligned}
$$

At the end, we got the exact quantities described below at $c=c_{r}$ :

$$
\begin{aligned}
C_{1}(0) & =\frac{i}{2 \omega_{0}}\left(g_{20} g_{11}-2\left|g_{11}\right|^{2}-\frac{1}{3}\left|g_{02}\right|^{2}+\frac{1}{2} g_{21}\right), \\
\mu_{2} & =-R_{e} C_{1}(0) / \alpha^{\prime}(0), \tau_{2}=-\frac{\mid m C_{1}(0)+\mu_{2} \omega_{0}^{\prime}(0)}{\omega_{0}}, \beta_{2}=2 R_{e} C_{1}(0),
\end{aligned}
$$


and the Marsden-MacCracken index is:

$$
\begin{aligned}
I_{M}= & \omega_{0}\left(F_{30}^{1}+F_{12}^{1}+F_{21}^{2}+F_{03}^{1}\right) \\
& +F_{20}^{1} F_{20}^{2}-F_{20}^{1} F_{11}^{1}+F_{20}^{2} F_{11}^{2} \\
& +F_{02}^{2} F_{11}^{2}-F_{02}^{1} F_{11}^{1}-F_{02}^{1} F_{02}^{2}
\end{aligned}
$$

\section{Results}

Considering that, system (1) undergoes a $\mathrm{HB}$ at equilibrium $S$ when parameter $c$ passes the critical value $c_{r}$, the following properties hold [24, 44]:

1. If $I_{M}>0$ and $\mu_{2}>0\left(I_{M}<0\right.$ and $\left.\mu_{2}<0\right)$, the Hopf bifurcation is non-degenerate and subcritical with unstable limit cycle (supercritical with stable limit cycle).

2. If $\beta_{2}<0\left(\beta_{2}>0\right)$, the bifurcating periodic solutions are orbitally stable (unstable).

3. If $\tau_{2}>0\left(\tau_{2}<0\right)$, the period of bifurcating periodic solutions increases (decreases).

Furthermore, the system has a unique amplitude solution of approximated period:

$T=\frac{2 \pi}{\omega_{0}}\left(1+\tau_{2} \varepsilon^{2}+0\left(\varepsilon^{4}\right)\right)$

and the characteristic exponent associated with this solution is:

$\beta=\beta_{2} \varepsilon^{2}+0\left(\varepsilon^{4}\right)$

where $\varepsilon^{2}=\frac{c-c_{r}}{\mu_{2}}+0\left(c-c_{r}\right)^{2}$ (with $\left.\mu_{2} \neq 0\right)$.

Referring to the development presented in [44], we deduce a mathematical approximation of the bifurcating periodic solution (except for an arbitrary phase angle):

$X=(x, y, z)^{T}=X_{0}\left(c_{r}\right)+P Y=\left(\begin{array}{l}x_{0}\left(c_{r}\right) \\ y_{0}\left(c_{r}\right) \\ z_{0}\left(c_{r}\right)\end{array}\right)+\left(\begin{array}{ccc}1 & 0 & -\frac{1}{\gamma} \\ a & \omega_{0} & 1 \\ \frac{c-\omega_{0}^{2}}{c} & \frac{a \omega_{0}}{c} & \frac{d}{a}\end{array}\right)\left(\begin{array}{c}\bar{x}_{2} \\ \bar{y}_{2} \\ \bar{z}_{2}\end{array}\right)$

Considering that, $\xi=\varepsilon \mathrm{e}^{\frac{2 \pi i \tau}{T}}+O(\varepsilon)$, we have $\bar{x}_{2}=\operatorname{Re} \xi=\varepsilon \cos \left(\frac{2 \pi}{T} \tau\right) ; \quad \bar{y}_{2}=\operatorname{Im} \xi=\varepsilon \sin \left(\frac{2 \pi}{T} \tau\right)$ and $\bar{z}_{2}=0+0(|\xi|)$.

We deduce therefore

$$
\left(\begin{array}{l}
x \\
y \\
z
\end{array}\right)=\left(\begin{array}{l}
x_{0}\left(c_{r}\right) \\
y_{0}\left(c_{r}\right) \\
z_{0}\left(c_{r}\right)
\end{array}\right)+\left(\begin{array}{c}
\varepsilon \cos \left(\frac{2 \pi \tau}{T}\right) \\
\varepsilon a \cos \left(\frac{2 \pi \tau}{T}\right)+\varepsilon \omega_{0} \sin \left(\frac{2 \pi \tau}{T}\right) \\
\varepsilon\left(1-\frac{\omega_{0}^{2}}{c_{r}}\right) \cos \left(\frac{2 \pi \tau}{T}\right)+\varepsilon \frac{a \omega_{0}}{c_{r}} \sin \left(\frac{2 \pi \tau}{T}\right)
\end{array}\right)+o(\varepsilon) .
$$

\subsection{Numerical results of Hopf bifurcation analysis}

Numerical calculations are done to demonstrate the above theoretical results on the Hopf bifurcation. For this instance, we take system (1) with the following parameter values $a=0.2, b=0.001, d=0.5$, from where we realize the unique non-trivial solution $S(7.2848,1.4569,7.2848)$. Using this equilibrium, the critical value of the bifurcation parameter $c$ is $c_{r}=0.468862270$. For this critical value, we have one real eigenvalue $\lambda_{3}\left(c_{r}\right)=-0.528979$ and two pure imaginary ones $\lambda_{1,2}\left(c_{r}\right)= \pm i 0.746657$.

Furthermore, $R_{e}\left(\lambda^{\prime}\left(c_{r}\right)\right)=-0.0982242<0$ and the condition for a HB is satisfied. Moreover, the traditional coefficients for the direction of the bifurcation are:

$g_{11}=-0.1393974946+0.2236314317 i$

$g_{02}=0.2630777742+0.01525658955 i$

$g_{20}=-0.1295450654-0.2294793124 i$

$g_{21}=-1.398529250+0.216574027 i$ and

$C_{1}(0)=-0.7012859617+0.046239916 i$.

Finally, we realise the principal results:

$\mu_{2}=46.60883592>0$;

$\beta_{2}=-1.402571923<0$;

$\tau_{2}=-58.42074455<0$ and $I_{M}=0.3155780951>0$.

Looking into the above results $\left(I_{M}>0\right.$ and $\left.\mu_{2}>0\right)$, it is obvious that the Hopf bifurcation is subcritical and nondegenerate. Hence, the equilibrium $O(0,0,0)$ of system (8) is stable when $c_{r}<c$ and the equilibrium loses its stability; hence, a Hopf bifurcation comes when $c$ decreases pass $c_{r}$. As $\beta_{2}<0$, the bifurcating periodic solutions are asymptotically stable orbits with a period given approximatively by:

$$
\begin{aligned}
T= & 8.410823176(1-1.253426381(c-0.4688622719)) \\
& +O(c-0.4688622719)^{2}
\end{aligned}
$$

and the corresponding characteristic exponent is

$\beta=-0.03009240405(c-0.4688622719)+O(c-0.4688622719)^{2}$

The bifurcating periodic solution is presented for $c \approx c_{r}$ in Fig. 2a, b, and we observe that it is orbitally stable. For $c_{1}<c_{r}$, Fig. $2 c$ illustrates that in the domain $\left[c_{1}, c_{r}[\right.$, the 

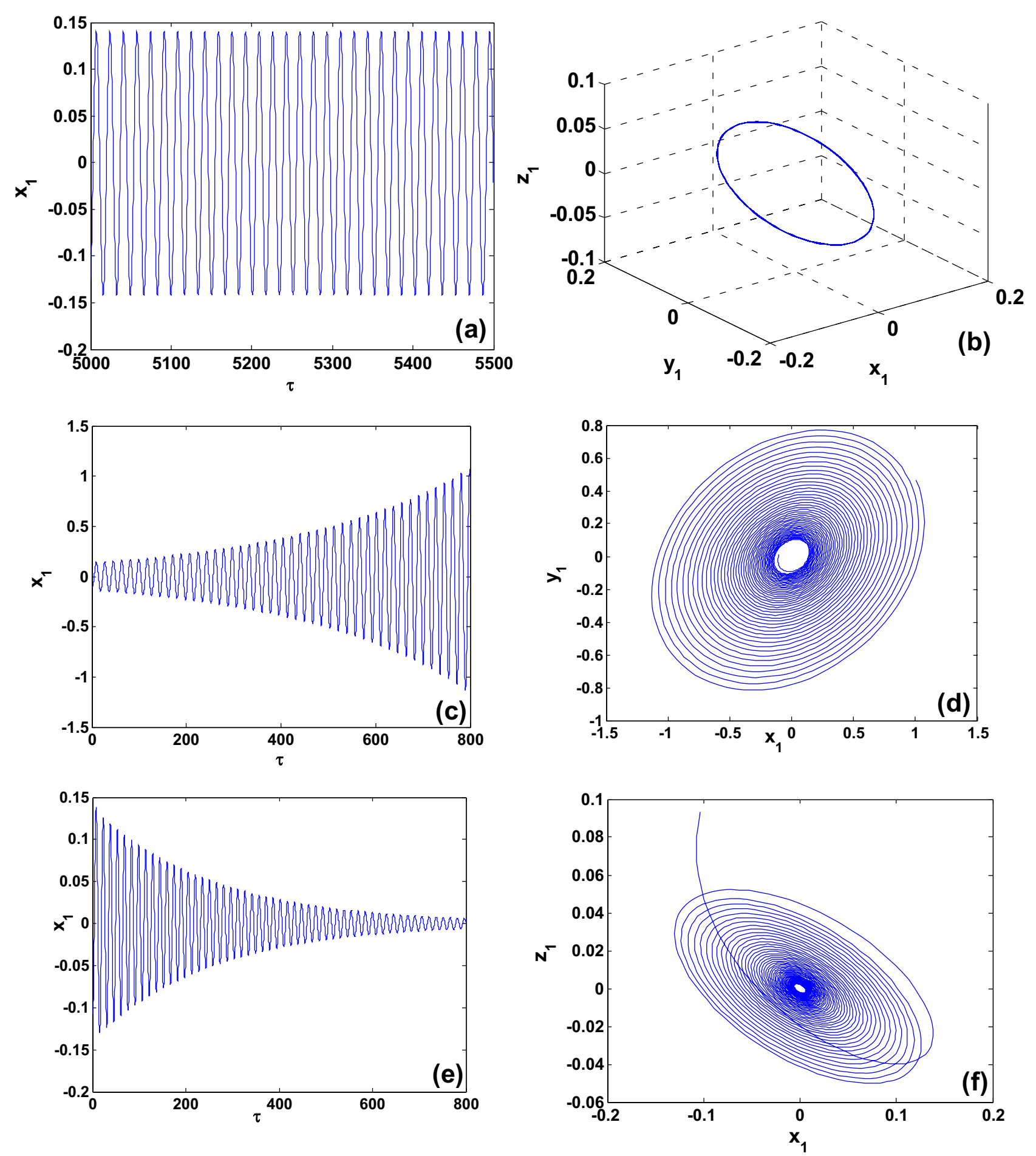

Fig. 2 Time series and phase portrait of system (4) with the initial condition $(-0.1,0.02,0.1)$ while keeping $a=0.2, b=0.001, d=0.5$. $\mathbf{a}$ and $\mathbf{b}$ for $c_{r}=0.468862 ; \mathbf{c}$ and $\mathbf{d}$ for $c_{1}=0.42<c_{r}$; e and $\mathbf{f}$ for $c_{2}=0.56>c_{r}$ 

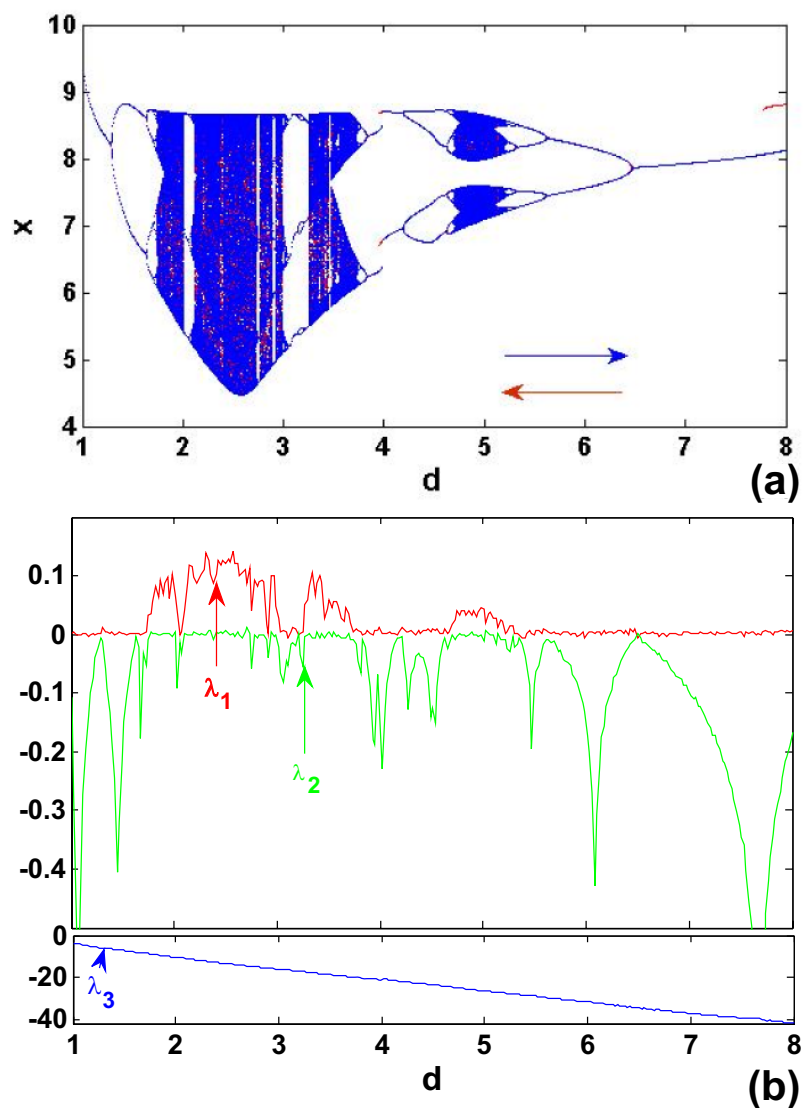

Fig. 3 Bifurcation diagram a showing local maxima of the coordinate $x$ of the attractor and corresponding graph of three largest Lyapunov exponents $\mathbf{b}$ versus parameter $d$ that is varied in tiny steps in the range $1 \leq d \leq 8$ with the initial condition $(-0.1,0.2,1.1)$ for $b=0.001, a=0.5, c=1$. The positive value of $\lambda_{1}$ is the signature of chaotic motion

origin is an unstable focus. Meanwhile, for $c_{2}>c_{r}$ in the domain $\left[c_{r}, c_{2}\right.$ [, Fig. 2e, $f$ shows that the origin is a stable focus surrounded by an unstable limit cycle for which the amplitude increases with $\sqrt{c_{2}-c_{r}}$.

The objective of this section was to present the mechanisms that give rise to the drain of stability generated by the disappearance or birth of a periodic orbit thus presenting the transition mechanism from a stationary regime to oscillations (or inverse). As a result of Hopf bifurcation (HB) study in the model, it is found that the volumes in the phase space are contracted if the real parts of the eigenvalues $(\lambda)$ are all negative and increased if they are positive. When the value of the control parameter decreases, from a critical value, the system becomes unstable; it has bifurcations that cause jump phenomena between the stable solutions.

\section{Dynamics of the oscillator}

\subsection{Bifurcation and route to chaos}

To examine various passages to chaos in the oscillator, system (1) is resolved numerically through the classical fourth-order Runge-Kutta integration formulas. Throughout this paper, the time grid is always fixed to $\Delta t=2 \times 10^{-3}$ and the calculations are done through variables and constants parameters in extended precision mode. To cancel the transient system (1) is integrated for a sufficiently long time with each group of parameters. The Lyapunov spectrum, bifurcation diagrams, frequency spectrum and phase portraits are used to investigate the sensibility of the system. When varying the parameter $d$ of the system (1), the bifurcation diagram of Fig. 3a displaying local maxima of the state vector $x$ and the equivalent graph of Lyapunov spectrum Fig. 3b of the model. Both figures are gotten by controlling parameter $d$ that is varied in tiny steps in the interval $1 \leq d \leq 8$ with the initial condition $(-0.1,0.2,1.1)$ for $b=0.001, a=0.5, c=1$. For these graphs, it can be seen that, when the parameter $d$ is varied, the model exhibits complex dynamical behaviours including: period-doubling route to chaos, reverse period doubling and chaos. The fragile level of chaos acts by the model is clearly proven by the low values of $\lambda_{\text {max }}$, that are always $\lambda_{\max }<0.20$. There is a satisfied concordance between the bifurcation diagram and the graph of Lyapunov exponents. Moreover, for Fig. 3a particularly, the bifurcation diagram in blue (respectively, in red) is obtained by increasing (respectively, decreasing) the control parameter $\mathrm{d}$ in this same interval using continuation technics. These strategies called upward and backward bifurcation is generally used to find windows in which the phenomenon of the coexistence of attractors occurs in the systems. For this range of parameter, we see that the two diagrams (blue and red) are perfectly superimposed, and thus, it shows that there is no coexistence of attractors; however, this phenomenon may appear for other range of parameters.

With some parameters settings in Fig. 3, various numerical phase portraits (Figs. 4a-c, 11, 12 and 13) and frequency spectrum (Fig. 4e) were produced. Those figures demonstrate various chaos mechanisms in the system for changing the parameter $a$, confirming different bifurcation sequences depicted in Figs. 3 and 6.

In addition, the complicated dynamics of the relatively simple chaotic system for $a=0.5$, are illustrated in Fig. 4 by ways of 3D phase portraits (Fig. $4 \mathrm{~d}$ ) and time series (Fig. 4f). 

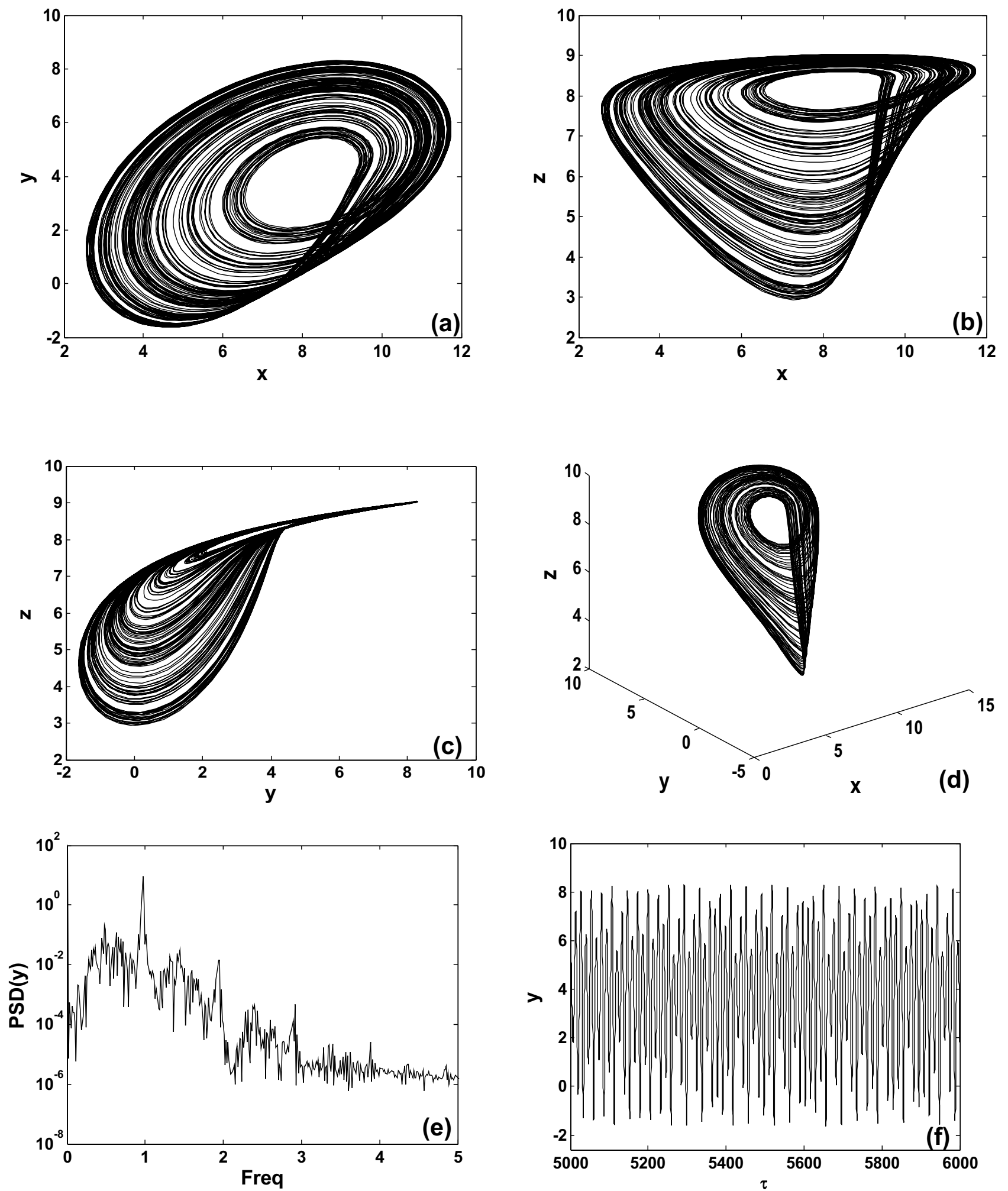

Fig. 4 Two-dimensional views a-c of the attractor projected, illustrating the complexity of the system, $\mathbf{d}$ the corresponding 3D projection of attractor; e the corresponding frequency spectra and the

corresponding time series $\mathbf{f}$ for $a=0.5, b=0.001, c=1, d=2$ with the initial condition $(-0.1,0.2,1.1)$ 

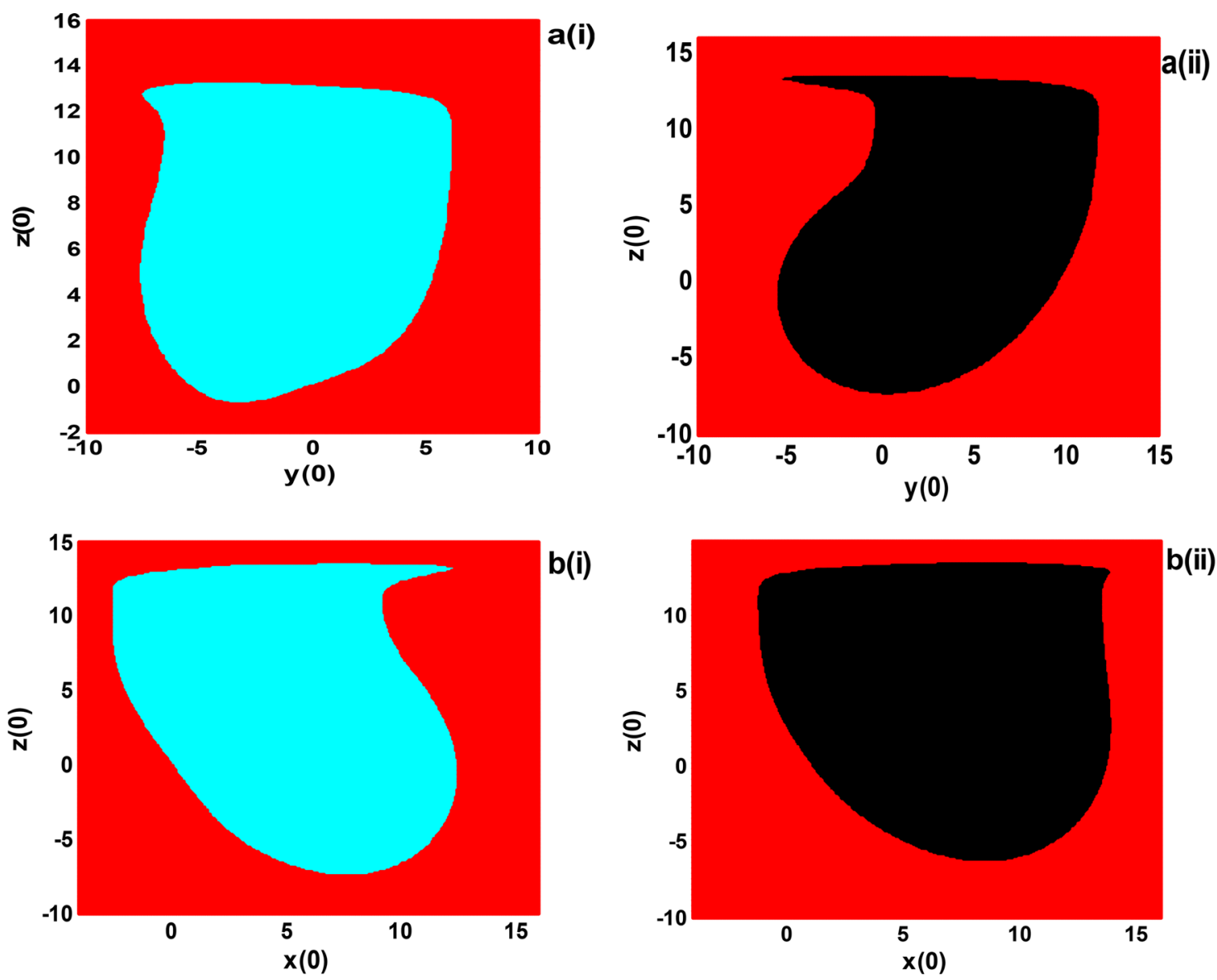

b(i)
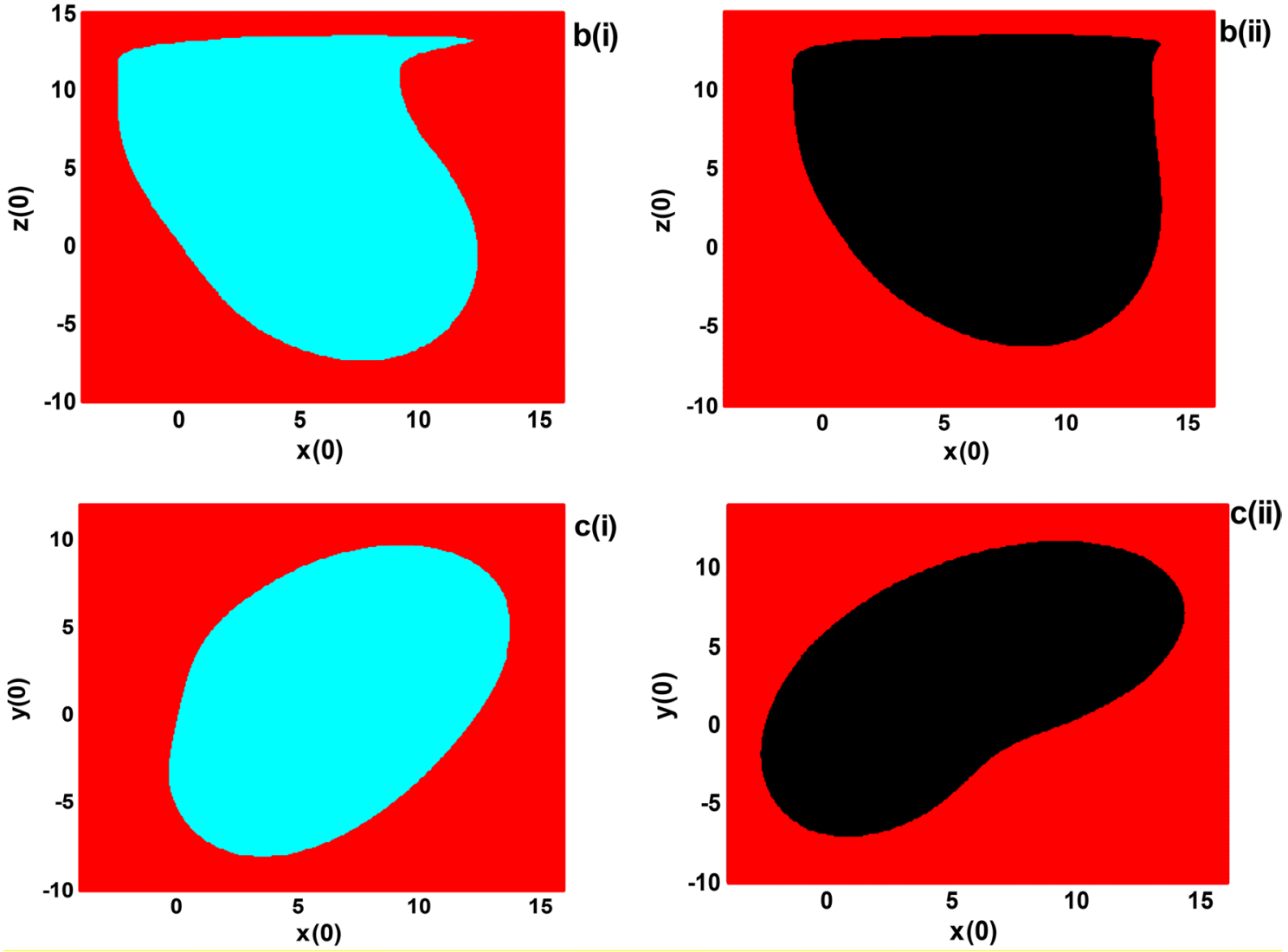

Fig. 5 Structure of the sections of the basin of attraction for $\mathbf{a}(\mathbf{i}) x(0)=0, \mathbf{b}(\mathbf{i}) y(0)=0, \mathbf{c}(\mathbf{i}) z(0)=0$ corresponding to the trivial fixed point and a(ii) $x(0)=8.3353 \mathbf{b}$ (ii) $y(0)=4.1677 \mathbf{c}$ (ii) $z(0)=8.3354$ corresponding to the non-trivial fixed point. These

cross sections are associated to the chaotic solutions and are shown in Fig. 3a-d; red zones correspond to unbounded solutions while cyan and black are associated chaos motion

According to [45], the Lyapunov dimension $\left(D_{\mathrm{L}}\right)$ of the attractor is figured out by using the formula of Kaplan and Yorke:

$D_{\mathrm{L}}=k+\frac{1}{\left|\lambda_{k+1}\right|} \sum_{j=1}^{k} \lambda_{j}$ 
(a)

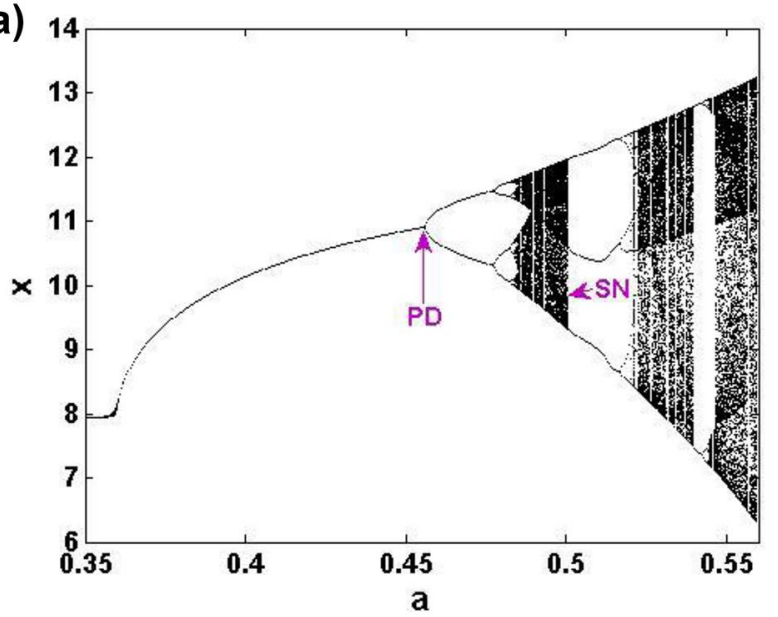

(b)

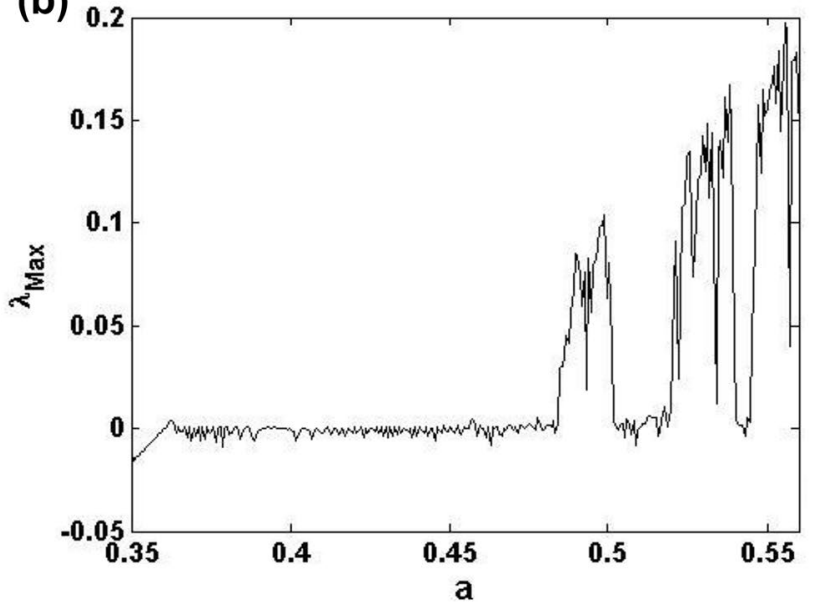

Fig. 6 Bifurcation diagram a showing local maxima of the coordinate $x(\tau)$ of the attractor and the corresponding 1D largest Lyapunov exponent $\left(\lambda_{\text {Max }}\right)$ in terms of the control parameter $a$ that is varied in tiny steps in the range $0.35 \leq a \leq 0.56$ with the initial condition $(-0.1,0.2,1.1)$ for $b=0.001, c=1, d=2$. We can easily observe the saddle-node bifurcation (SN) and period-doubling (PD) phenomenon

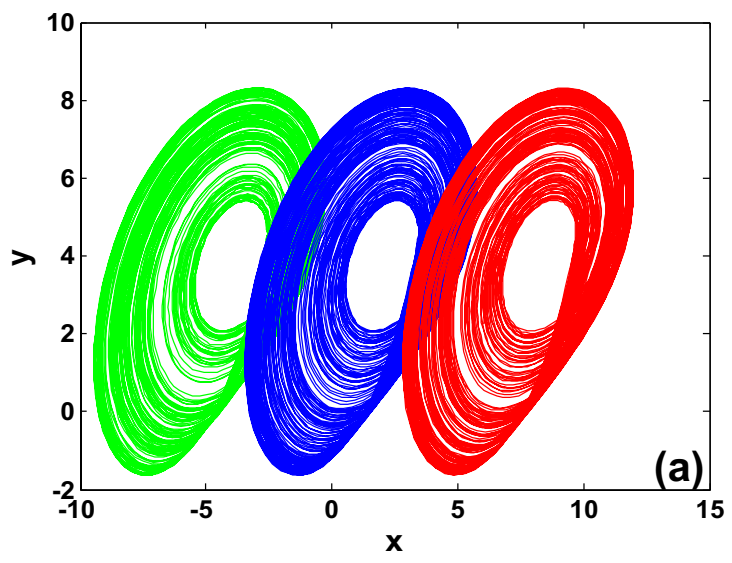

where $k$ satisfies $\sum_{j=1}^{k} \lambda_{j} \geq 0$ and $\sum_{j=1}^{k+1} \lambda_{j}<0$; the Lyapunov exponents being arranged such that $\lambda_{j} \geq \lambda_{j+1}$. This chaotic attractor has the following Lyapunov exponent spectrum: $\lambda_{1}=0.140, \lambda_{2}=0.000, \lambda_{3}=-12.32$, the corresponding Kaplan-Yorke dimension being $D_{\mathrm{L}}=2.011$. Since $2<D_{\mathrm{L}}<3$, the model exhibited a strange attractor [46]. Considering the range of parameter in Fig. 4 where the system has a chaotic behaviour, we obtain two equilibrium points, one trivial $O(0,0,0)$ whose eigenvalues are $\lambda_{1}=0.3389$ and $\lambda_{2,3}=0.0796 \pm 1.7143 i$, and the other non-trivial $S(7.2848,1.457,7.2848)$ where the eigenvalues are $\lambda_{1}=-8.0938$ and $\lambda_{2,3}=0.1282 \pm 0.9435 i$ each corresponding to unstable foci. In order to be able to determine the nature of these attractors, we have computed the basins of attraction of Fig. 5 where the zones in red correspond to unbounded solutions, while cyan and black are associated chaos motion. Based these figures, we find that the trivial equilibrium point is localized at the boundary between chaotic region and the unbounded region (see Fig. $5 a(i)-c(i)$ ), while the non-trivial equilibrium point is well contained in the bounded (chaotic) region (see Fig. 5a(ii)-c(ii)); this allows us to conclude that the attractors shown in Fig. 4 are self-excited $[47,48]$. Furthermore, the influence of the manipulating parameter $a$ on the dynamics of the system is presented through the bifurcation diagram of Fig. $6 a$ and the corresponding graph of Lyapunov exponent of Fig. 6b. Period-doubling bifurcation (PD), periodic window phenomena and saddle-node bifurcation (SN) are easily observed. From Fig. 6b, it can be seen that the quality of the largest Lyapunov exponent curve has been plotted by taking a sufficiently large simulation time to ensure that when the system is not chaotic, this exponent is close to zero. One can also notice that at the beginning of this diagram, this LE is slightly less than zero, which is normal because at this moment the system does

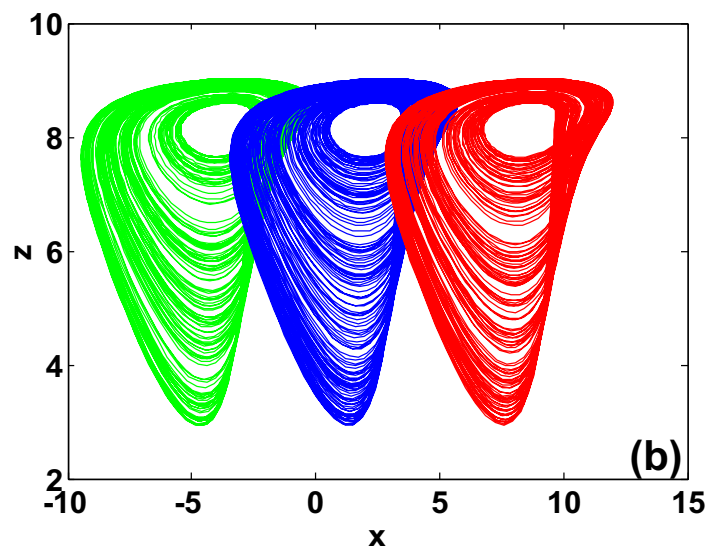

Fig. 7 Offset boosting of the chaotic attractor for varying the control parameter $k$ : $\mathbf{a}$ in $x-y$ plane, and $\mathbf{b}$ in $x-z$ plane for $k=-0.25$ (green) $k=6$ (blue), and $k=12$ (red) 
(a)

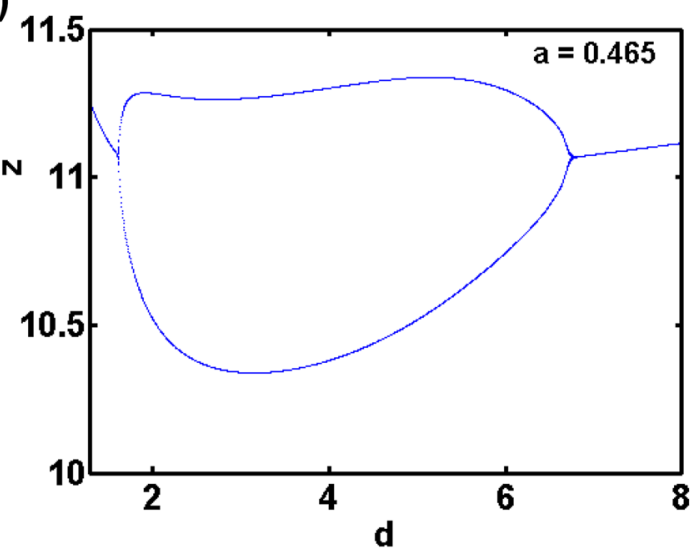

(c)

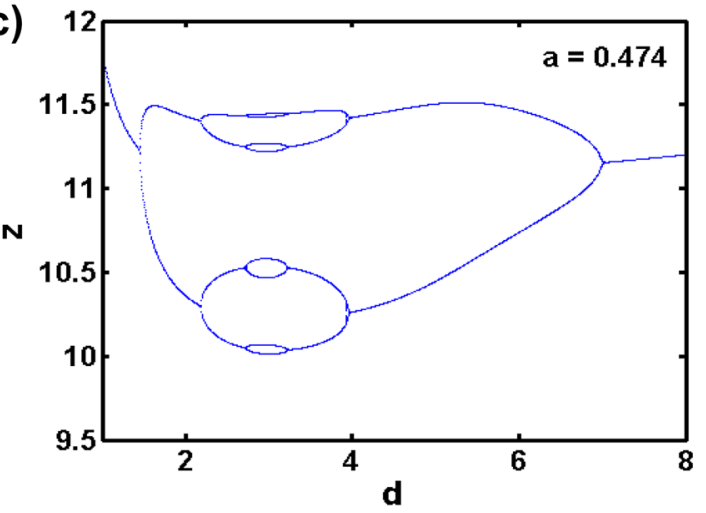

(e)

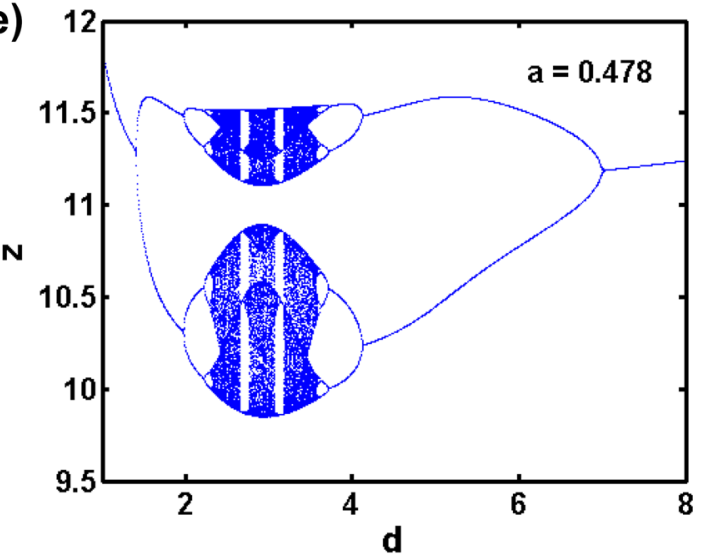

(b)

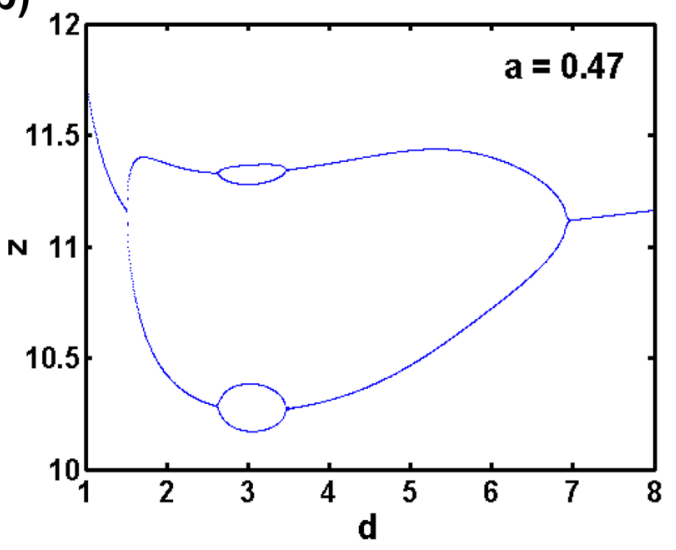

(d)

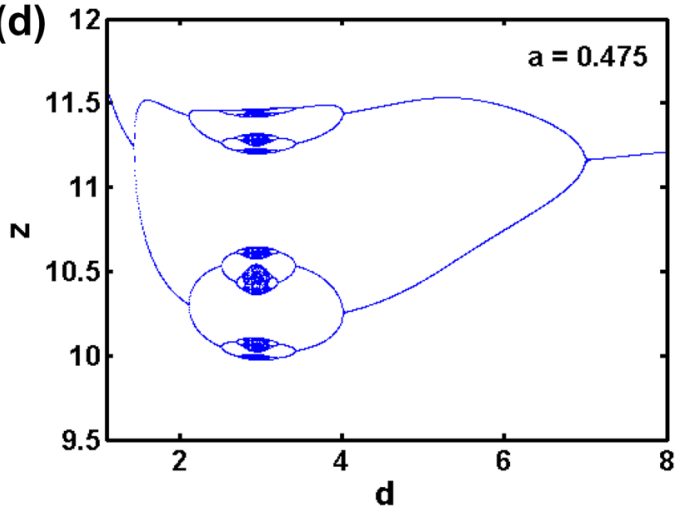

(f)

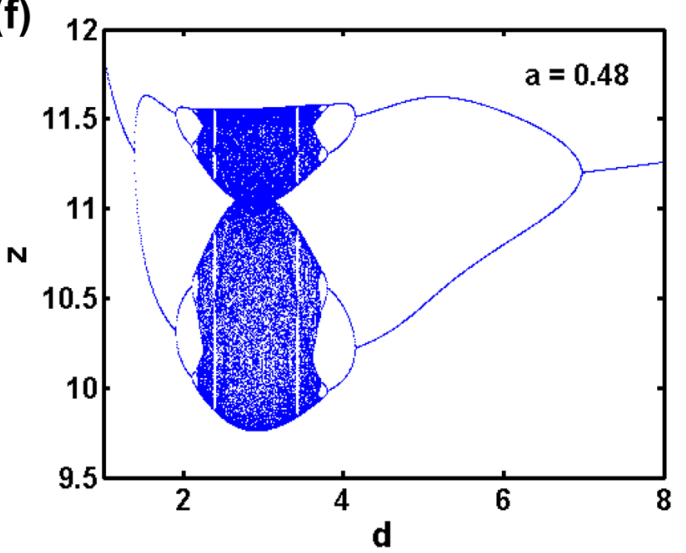

Fig. 8 Bifurcation diagrams showing local maxima of the coordinate $z$ in terms of the parameter $d$ for remerging Feigenbaum tree (bubbling): primary bubble in (a); period-4 bubble in (b); period-8 bubble in (c); full Feigenbaum remerging tree at (d); (e) and (f), respectively

not yet oscillate and the corresponding phase portrait is a fixed point.

\subsection{Offset-boosting behaviour}

In practical, the chance of amplitude control represents an attractive property of a potential chaos generator
[49-52]. Therefore, the introduced feedback state stands for a useful alternative to control the amplitude of the variables. For our system (1), the variables $x$ and $y$ appear without the nonlinearity; thus, they can be well controlled. Also, the variable $x$ is offset-boosted by the changes $x \rightarrow x+k$, where $k$ represents a constant (note 
Fig. 9 Electronic circuit realization of the autonomous chaotic system with exponential nonlinearity

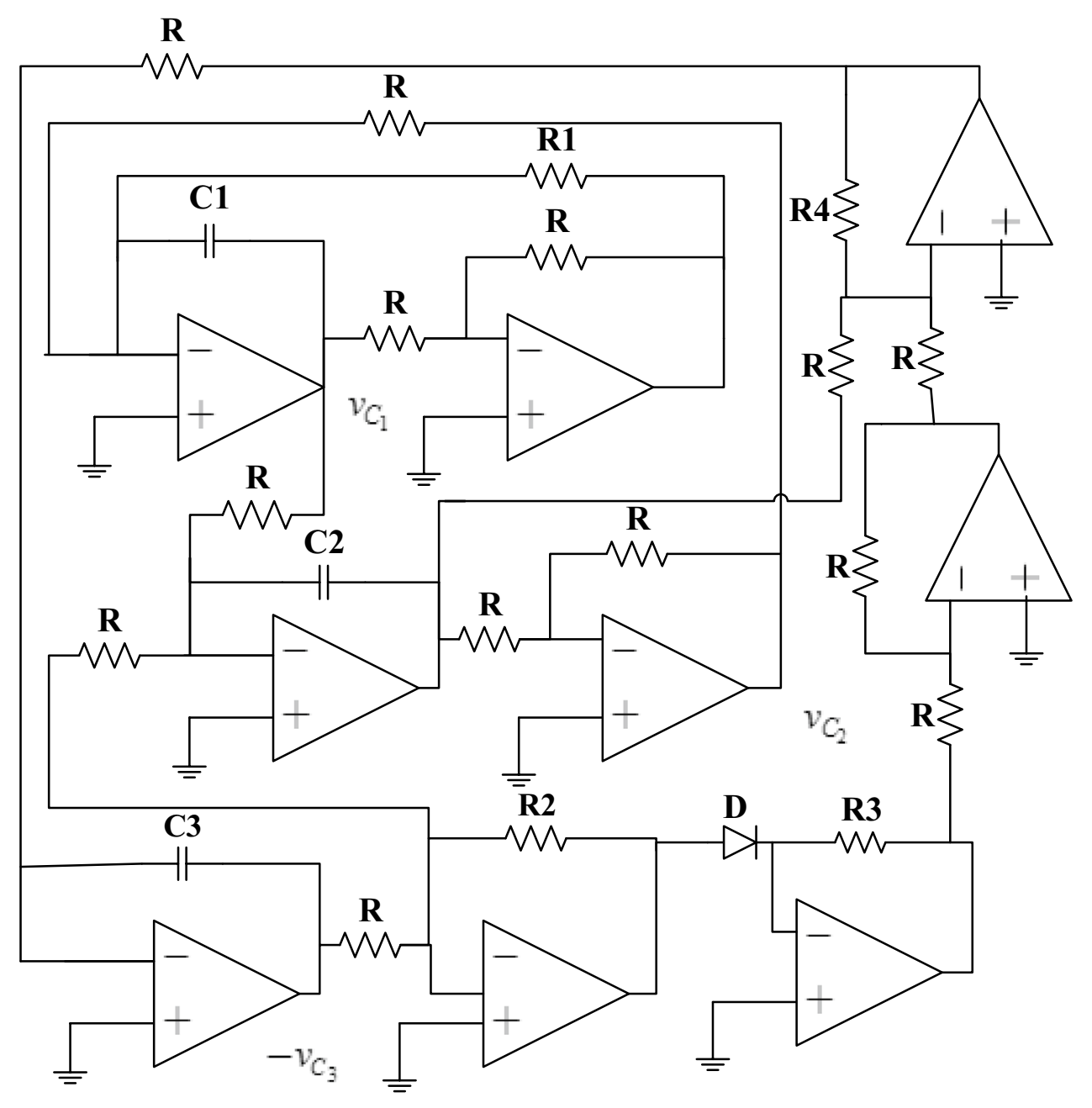

Table 2 Values of electronics components used for the laboratory experimental study

\begin{tabular}{lll}
\hline Parameter & Signification & Value \\
\hline$C_{i}(i=1,2,3)$ & Capacitance & $10 \mathrm{nF}$ \\
$R$ & Resistance & $10 \mathrm{k} \Omega$ \\
$R_{1}$ & Resistance & Tuneable \\
$R_{2}$ & Resistance & $494 \Omega$ \\
$R_{3}$ & Resistance & $372856,077 \Omega$ \\
$R_{4}$ & Resistance & $20 \mathrm{k} \Omega$ \\
$D$ & 1N4148 diode & $I_{S}=2.682 \mathrm{nA}$, \\
& & $n=1.9$, \\
& & $V_{T}=26 \mathrm{mV}$ \\
\hline
\end{tabular}

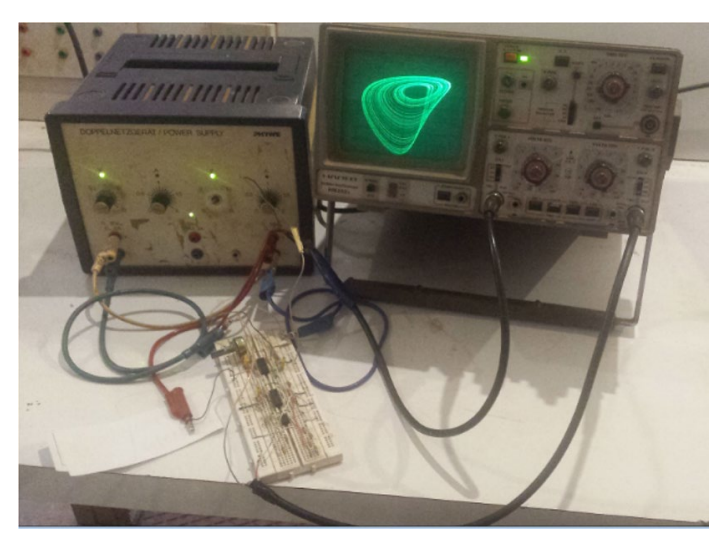

Fig. 10 The experimental chaotic circuit in operation 

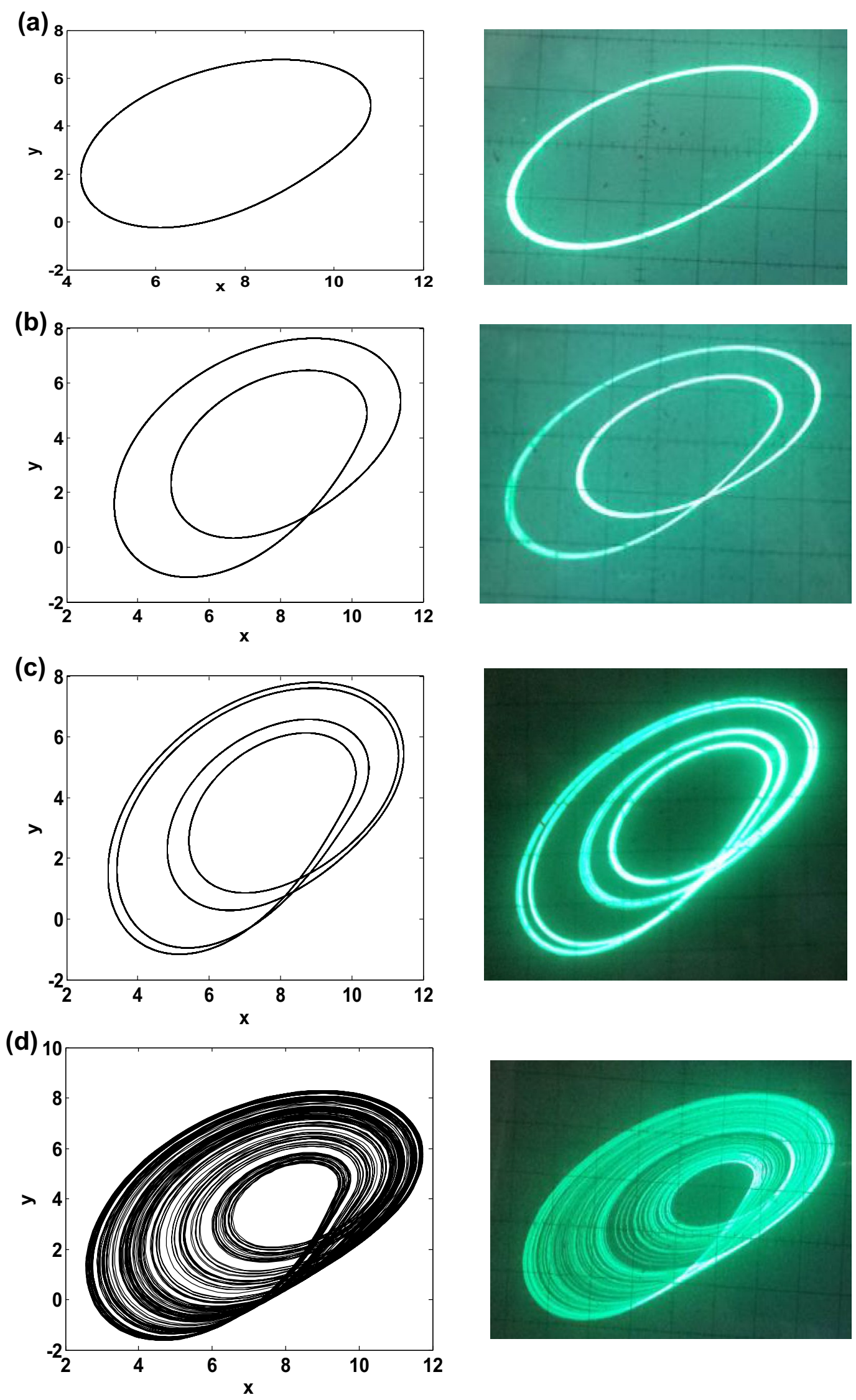
4Fig. 11 Experimental phase portraits (right) obtained from the circuit using a dual-trace oscilloscope in the $X Y$ mode; the corresponding numerical phase portraits are shown in the left. Output voltages $v_{c_{1}}$ and $v_{c_{2}}$ (are fed to the $X$ and $Y$ input, respectively: a period- 1 for $R_{1}=22222.3 \Omega$ (i.e. $a=0.45$ ); $\mathbf{b}$ period-2 for $R_{1}=21276.6 \Omega$ (i.e. $a=0.47$ ); c period- 4 for $R_{1}=11,620$ (i.e. $a=0.48$ ); d single-band chaos for $R_{1}=20 \mathrm{k} \Omega$ (i.e. $a=0.5$ ); (with $b=0.001$, $c=1, d=2$, the initial condition $(-0.1,0.2,1.1)$ for the numerical results). The scales are $X=50 \mathrm{mV} / \mathrm{div}$ and $Y=0.1 \mathrm{~V} /$ div for all pictures

that the offset boosting can equally be made with the state variable $y$ ).

The system (1) can hence be written as seen in Eq. 22 below.

$\left\{\begin{array}{l}\dot{x}=a(x+k)-y \\ \dot{y}=c((x+k)-z) \\ \dot{z}=d\left[y-b\left(e^{z}-1\right)\right]\end{array}\right.$

In Fig. 7, we have the offset boosting of the chaotic attractor for changing the manipulated parameter $k$.

\subsection{Remerging Feigenbaum and antimonotonicity}

An important characteristic of the model is antimonotonicity phenomenon or reverse period-doubling bifurcation. More so, in multiple nonlinear systems, periodic orbits can appear and then disappear when two bifurcation parameters are varied [53]. This particular scenario has been found in several problems in varied fields of science such as Chua circuit [54], Duffing oscillator [55], van der pol oscillator [56], hyperjerk system [57] and many other classes of nonlinear systems. According to [53] and [58], the first condition to observe the occurrence of reverse period-doubling bifurcation is the availability of periodic islands in the parameters space. In addition to this condition, the second one was that the model examined must remain unchanged due to the transformation $(x, y, z) \leftrightarrow(-x,-y,-z)$ where $(x, y, z)$ denotes the state variable. However, our model does not vary with respect to the above transformation, but the phenomenon of antimonotonicity does appear as illustrated in Fig. 3a. As concluded by $[47,48]$, the above condition for the occurrence of antimonotonicity in a nonlinear system is not always necessary. Figure 8 shows most bifurcation diagrams when the parameter $d$ is changed in the interval $1 \leq d \leq 8$ for some specific values of parameter $a$; thus, sample results have produced: (a) primary bubble for $a=0.465$; (b) period- 4 bubble for $a=0.47$; (c) period-8 bubble for $a=0.474$; (d), (e), (f) full Feigenbaum remerging tree. The values of the former parameters are $c=1, b=0.001$ with initial condition $(x(0), y(0), z(0))$ corresponding to $(-0.1,0.2,1.1)$.

\section{Experimental study}

The aim in this section is to construct and implement a specific analogue simulator $[59,60]$ for the dynamical examination of the model defined in system (1) to check the numerical/theoretical results obtained previously. To illustrate the chaos mechanism in the system, an experimental analysis of the simple chaotic system has been carried out. To better appreciate our results, numerical and experimental phase portraits are equivalent.

\subsection{Design of the analogue simulator}

The circuits consist of three channels to build up the integration of the three state variables $x, y$ and $z$, respectively. In the electronic circuit of Fig. 9, the operational amplifiers TL084 and associated circuitry perform the basic operations of addition, subtraction and integration; the nonlinear term of system (1) is implemented with the $1 \mathrm{~N} 4148$ diode. The current flowing across the diode as function of voltage between anode and cathode is described by [61, 62]:

$i_{D}=I_{\mathrm{s}}\left(\exp \left(\frac{V}{n V_{\mathrm{T}}}\right)-1\right)$,

where $I_{s} n$ and $V_{\mathrm{T}}$ indicate the reverse saturation current, emission coefficient and thermal voltage of the diode, respectively. Their values are $I_{\mathrm{s}}=2.682 \mathrm{nA}, n=1.9$ and $V_{\mathrm{T}}=26 \mathrm{mV}$.

Adopting a suitable (standard) time scaling, the simulator outputs can be visible on an oscilloscope by connecting the output voltage of $v_{c_{1}}$ to the $X$ input and the output voltage of $v_{c_{2}}$ to the $Y$ input for example. By applying the Kirchhoff's current and voltage laws to the electronic 
(a)

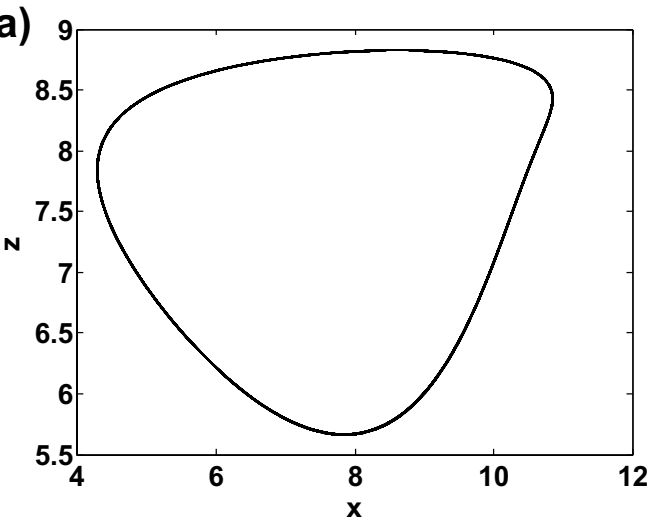

(b)

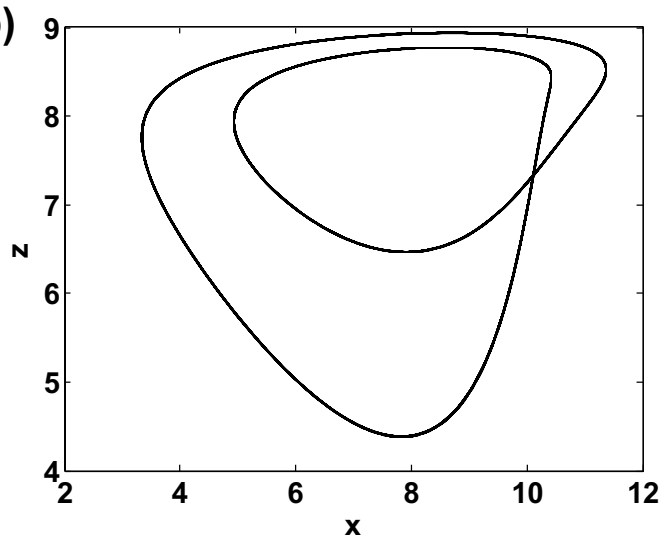

(c)

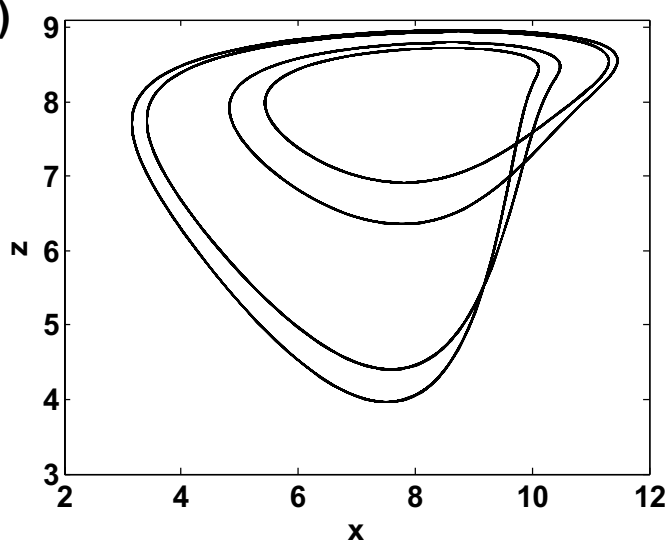

(d)

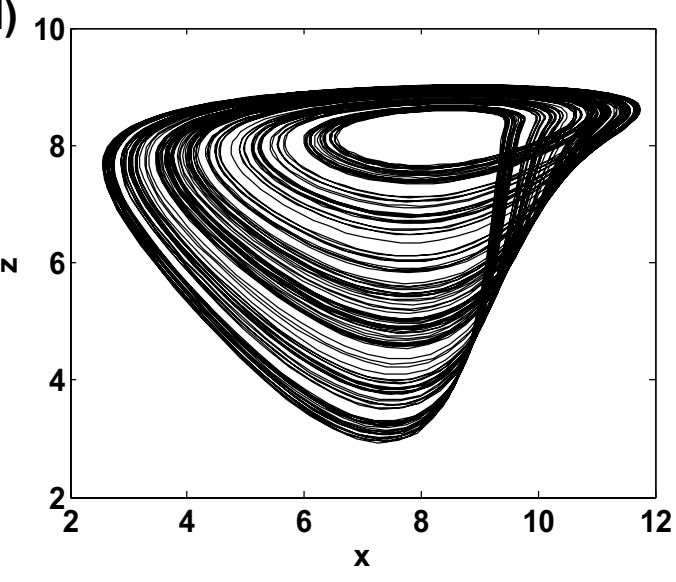

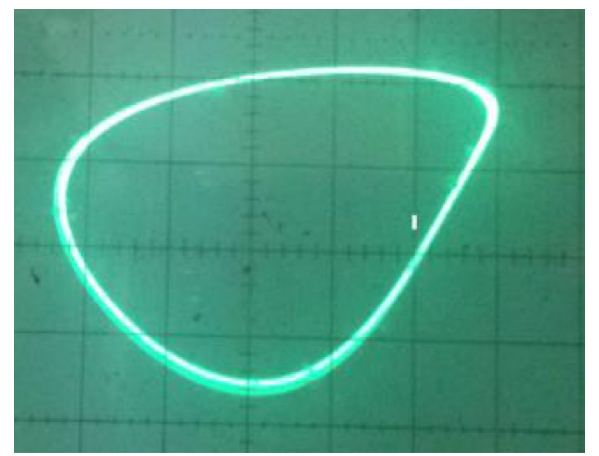
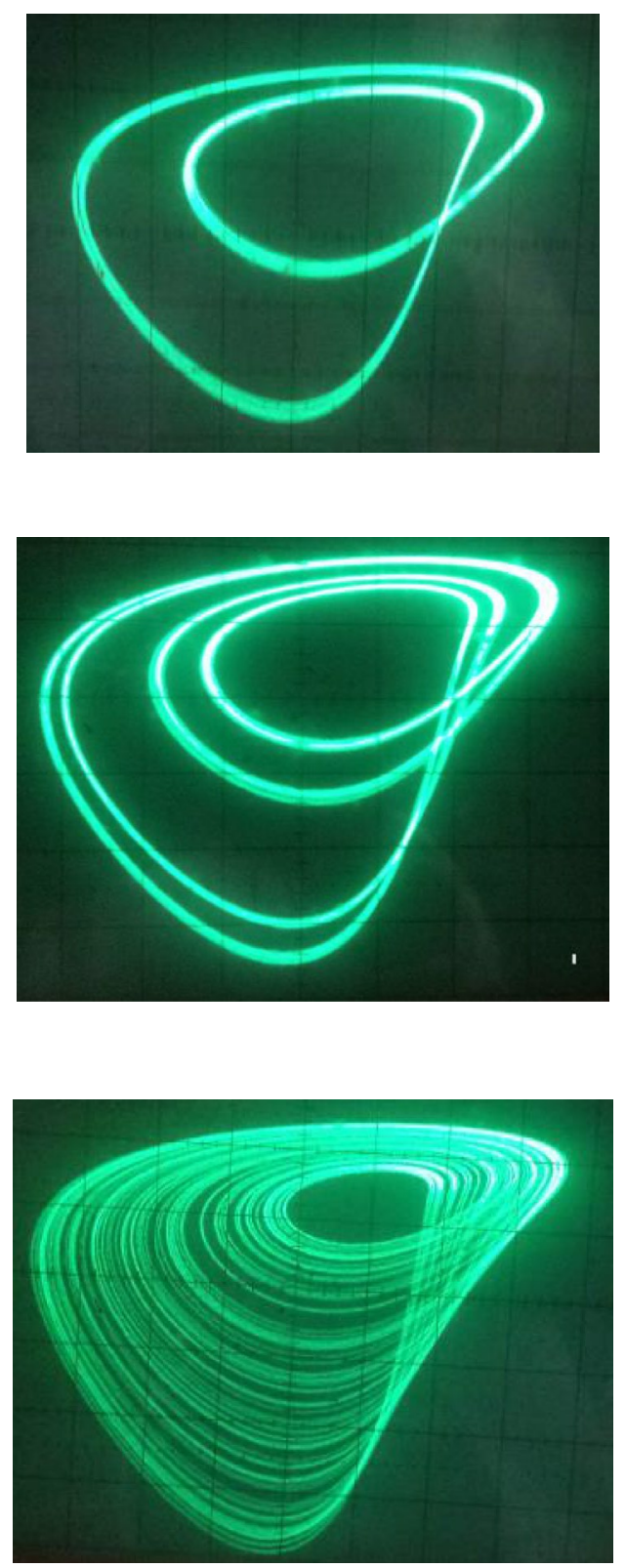
4Fig. 12 Experimental phase portraits (right) obtained from the circuit using a dual-trace oscilloscope in the $X Y$ mode; the corresponding numerical phase portraits are shown in the left. Output voltages $v_{c_{1}}$ and $v_{c_{3}}$ (are fed to the $X$ and $Y$ input, respectively: a period- 1 for $R_{1}=22222.3 \Omega$ (i.e. $a=0.45$ ); b period-2 for $R_{1}=21276.6 \Omega$ (i.e. $a=0.47$ ); c period- 4 for $R_{1}=11,620$ (i.e. $a=0.48$ ); d single-band chaos for $R_{1}=20 k \Omega$ (i.e. $a=0.5$ ); (with $b=0.001$, $c=1, d=2$, the initial condition $(-0.1,0.2,1.1)$ for the numerical results). The scales are $X=50 \mathrm{mV} / \mathrm{div}$ and $Y=50 \mathrm{mV} / \mathrm{div}$ for all pictures

circuit in Fig. 9; their circuital equations are derived in the following form:

$$
\left\{\begin{array}{l}
C_{1} \frac{\mathrm{d} v_{C_{1}}}{\mathrm{~d} t}=\frac{v_{C_{1}}}{R_{1}}-\frac{v_{C_{2}}}{R} \\
C_{2} \frac{\mathrm{d} v_{C_{2}}}{\mathrm{~d} t}=\frac{v_{C_{1}}}{R}-\frac{v_{C_{3}}}{R} \\
C_{3} \frac{\mathrm{d} v_{C_{3}}}{\mathrm{~d} t}=\frac{R_{4} v_{C_{2}}}{R}-\frac{R_{4} R_{3} I_{s}}{R}\left(\exp \left(\frac{R_{2} v_{C_{3}}}{R n v_{T}}\right)-1\right)
\end{array}\right.
$$

where $v_{C_{1}}, v_{C_{2}}$ and $v_{C_{3}}$, which represent the states $x, y$ and $z$, denote the voltages through the capacitors $C_{1}, C_{2}$ and $C_{3}$, respectively.

Setting $C_{1}=C_{2}=C_{3}=C$, and adopting a time unit of $10^{-4} s$, the parameters of system (1) are explicit in terms of figures of current, capacitor and resistors as follows:

$a=\frac{1}{10^{4} R_{1} C}, b=R_{3} I_{s}, c=\frac{1}{10^{4} R C}$ and $d=\frac{R_{4}}{10^{4} R^{2} C}$.

In short, the time-scaling procedure provides to the analogue devices the chances to work out under their bandwidth. It equally provides the chances to simulate the behaviour of the system at all frequency by determining the real-time $\tau$ versus the analogue calculation time $t$ ( $t=10^{-n} \tau$, where $n$ is positive integer relies on the values of resistors and capacitors used in the analogue computer (Fig. 9)).

\subsection{Experimental results}

Now we concentrate on the experimental study of our model with the aid of the electronic circuit. The effects of parameter $a$ on the conduction of the joint system are look into by monitoring a unique resistor $\left(R_{1}\right)$ while maintaining the other electronic component values constant (see Table 2). A practical implementation of Fig. 9 is figured out with the help of op. amplifiers (TL084CN), diode (1N4148), high-precision resistors and capacitors with corresponding values fixed as above. The bias is used by a 14VDC symmetric voltage source. Note that, when controlling the resistors $R_{1}$, it is discovered that the electronic simulator goes through a rich and spectacular dynamical behaviour admitting period-doubling route to chaos and chaos. Some sample phase portraits obtained experimentally are shown in Figs. 10, 11, 12 and 13. It is obvious that, they are alike similarity between the numerically computed phase portraits (left) and the experimental ones (right). Again, from Figs. 11, 12 and 13, one can clearly say that the experimental circuit can go through the same bifurcation scenarios as those obtained numerically.

The outputs gotten above in this test confirm that the analogue simulator is a strong equipment for the investigation of complex nonlinear systems.

\section{Conclusion}

In conclusion, a relatively simple 3D system with exponential nonlinearity has been studied in this paper. The basic dynamical analysis has highlighted complex and captivating phenomena in the model including period doubling, saddle-node bifurcation, various periodic windows, offset boosting, reverse period doubling and antimonotonicity. As one of the principal results of this works, the finding of a non-degenerate Hopf bifurcation happens in our system, when an appropriately chosen bifurcation parameter varies and arrives in its critical value. Furthermore, the direction of the Hopf bifurcation and stability of the bifurcating periodic solutions are break down into full meaning by enforce the Hassard algorithm. Hence, we use the Marsden and MacCracken index to show that this Hopf bifurcation at the equilibrium is non-degenerated. An electronic circuit including the diode nonlinear was built and employ to validate the obtained results. Laboratory experience results have given a good accord with the theoretical analysis. Due to its relative simplicity, the 3D autonomous system taken into this framework represents a reliable and palpable resource for generating a large diversity of nonlinear phenomena including antimonotonicity and the offset boosting. More interestingly, the model is capable for chaos-based engineering applications or it is applied for educational purposes. Finally, the detailed exploration of the parameter space in view of tracking all regions in 
(a)

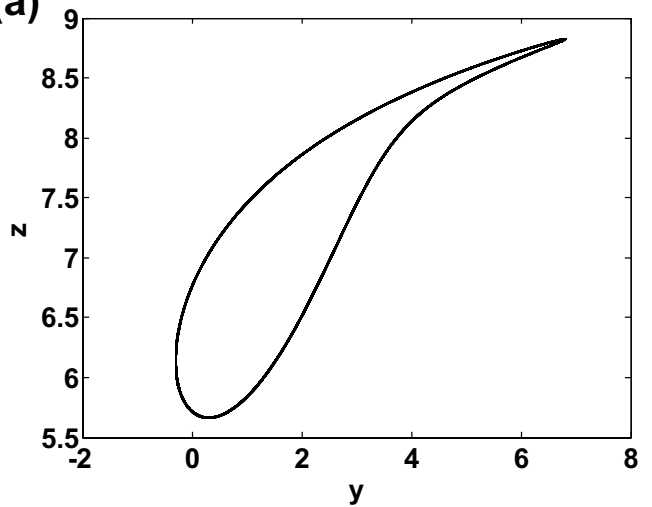

(b)

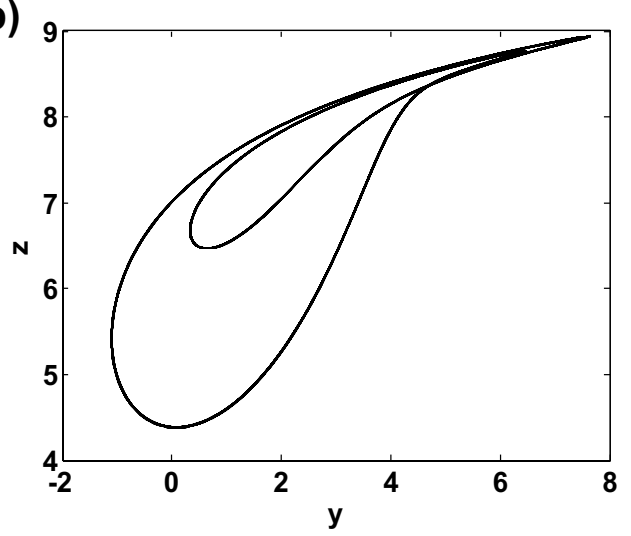

(c)

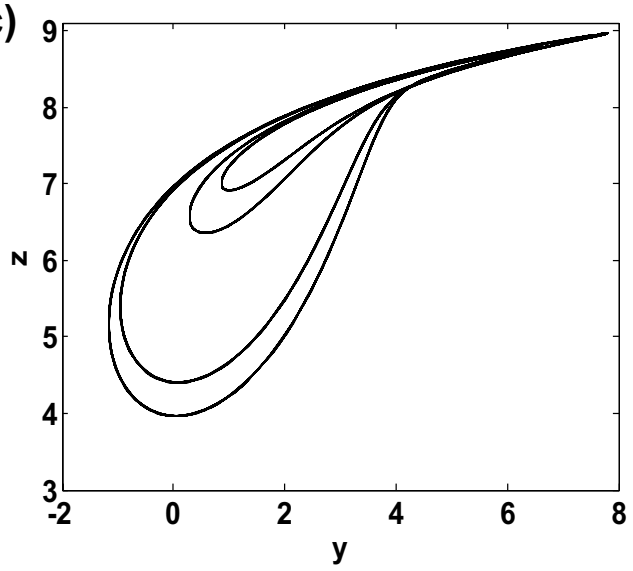

(d)

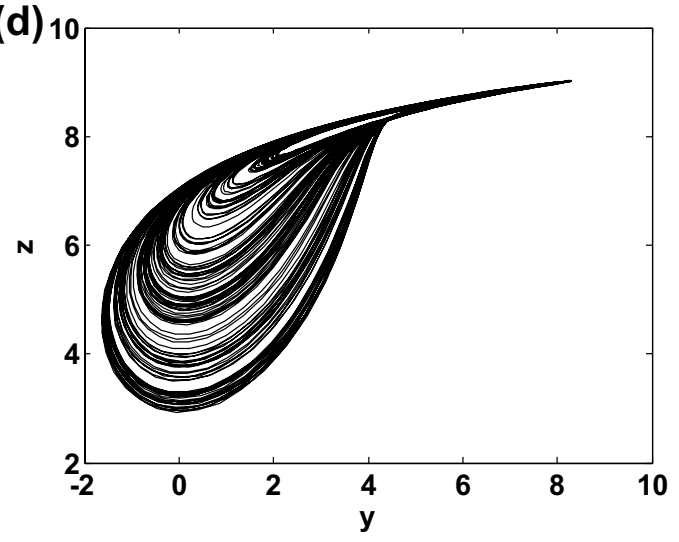

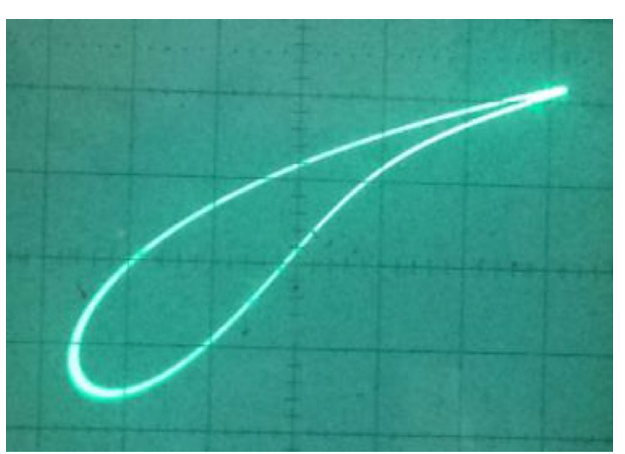
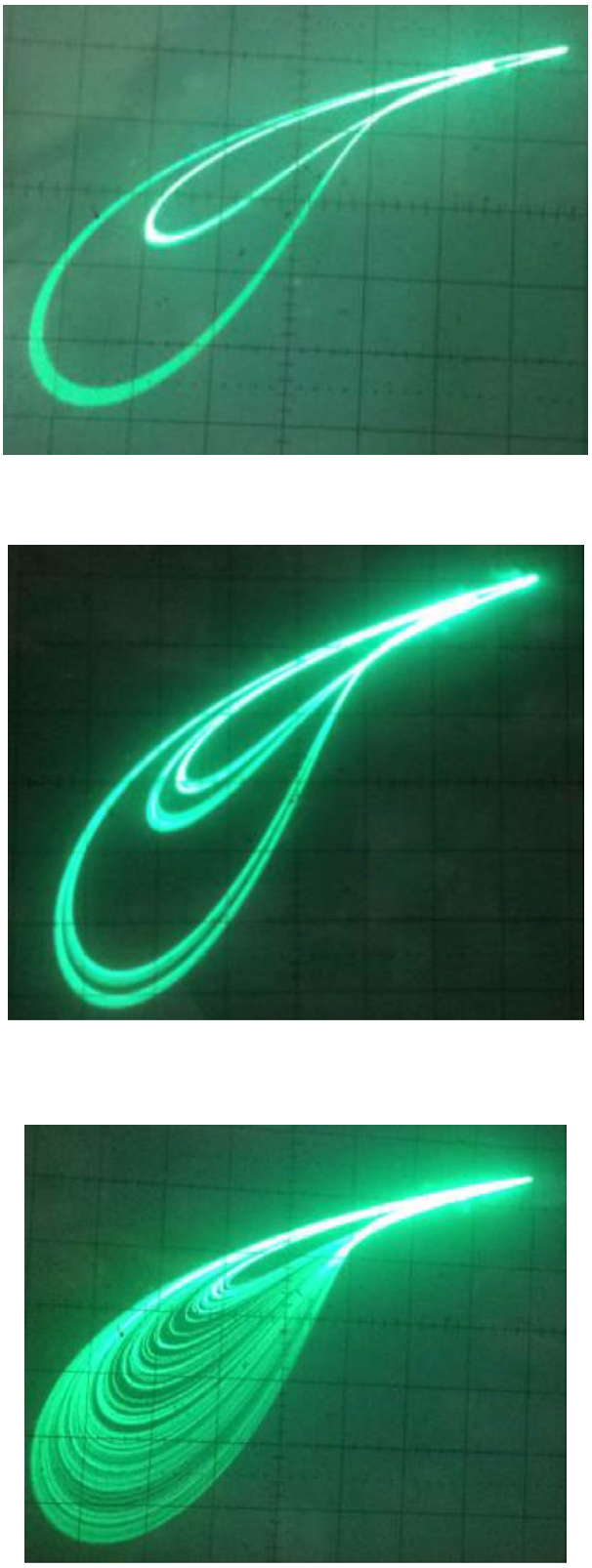
4Fig. 13 Experimental phase portraits (right) obtained from the circuit using a dual-trace oscilloscope in the $X Y$ mode; the corresponding numerical phase portraits are shown in the left. Output voltages $v_{c_{2}}$ and $v_{c_{3}}$ (are fed to the $X$ and $Y$ input, respectively: a period- 1 for $R_{1}=22222.3 \Omega$ (i.e. $a=0.45$ ); $\mathbf{b}$ period-2 for $R_{1}=21276.6 \Omega$ (i.e. $a=0.47$ ); c period- 4 for $R_{1}=11,620$ (i.e. $a=0.48$ ); d single-band chaos for $R_{1}=20 \mathrm{k} \Omega$ (i.e. $a=0.5$ ); (with $b=0.001$, $c=1, d=2$, the initial condition $(-0.1,0.2,1.1)$ for the numerical results). The scales are $X=50 \mathrm{mV} / \mathrm{div}$ and $Y=50 \mathrm{mV} / \mathrm{div}$ for all pictures

which the phenomenon of coexistence of multiple attractors occurs deserves further studies.

\section{Compliance with ethical standards}

Conflict of interest On behalf of all authors, the corresponding author states that there is no conflict of interest.

\section{Appendix: Some mathematical expressions}

Some mathematical expressions obtained during calculus are investigated here.

$$
\begin{aligned}
& A_{1}=\omega_{0}\left(\frac{-\left(a^{2}-c d\right)(a-c)}{c a}-\frac{a\left(1-\left(c-\omega_{0}^{2}\right)\right)}{\gamma c}+\frac{d c}{\gamma}\right) \\
& B_{1}=\omega_{0}^{2}\left(\frac{\left(a^{2}-c d\right)}{c a}+\frac{a^{2}}{\gamma c}+\frac{d}{\gamma}\right) \\
& C_{1}=\omega_{0}\left(\frac{d}{\gamma}+\frac{\left(a^{2}-c d\right)\left(\frac{a}{\gamma}+1\right)}{c a}+\frac{\left(\frac{a}{\gamma}+d\right)}{\gamma}\right) \\
& A_{2}=\frac{\left(a c-a \omega_{0}^{2}-c^{2} d\right)(a-c)}{c a} \\
& +\frac{\left(a c-a \omega_{0}^{2}+c d \gamma\right)\left(1-\frac{c-\omega_{0}^{2}}{c}\right)}{\gamma a}-\frac{(\gamma+c) d c}{\gamma} \\
& B_{2}=\omega_{0}\left(\frac{\left(a c-a \omega_{0}^{2}-c^{2} d\right)}{c a}-\frac{\left(a c-a \omega_{0}^{2}+c d \gamma\right)}{\gamma c}-\frac{(\gamma+c) d}{\gamma}\right) \\
& C_{2}=\frac{\left(a \omega_{0}^{2}+c^{2} d-a c\right)\left(\frac{a}{\gamma}+1\right)}{c a} \\
& +\frac{\left(a \omega_{0}^{2}-c d \gamma-a c\right)\left(\frac{1}{\gamma}+\frac{d}{a}\right)}{\gamma a}-\frac{(\gamma+c) d}{\gamma}
\end{aligned}
$$

$A_{3}=\frac{\omega_{0}\left(a c+\omega_{0}^{2}-c\right)(a-c)}{c}-a \omega_{0}\left(1-\frac{c-\omega_{0}^{2}}{c}\right)+\omega_{0} d c$

$B_{3}=\omega_{0}^{2}\left(\frac{\left(a c+\omega_{0}^{2}-c\right)}{c}+\frac{a^{2}}{c}+d\right)$

$C_{3}=\omega_{0}\left(\frac{\left(a c+\omega_{0}^{2}-c\right)\left(-\frac{a}{\gamma}-1\right)}{c}+\frac{a}{\gamma}+2 d\right)$

$M=\frac{c \gamma a}{\omega_{0}\left(a^{2} c+a^{2} \gamma+a \omega_{0}^{2}-c d \gamma-a c\right)}$

\section{References}

1. Çavuşoğlu Ü, Panahi S, Akgul A, Jafari S, kaçar S (2019) A new chaotic system with hidden attractor and its engineering applications: analog circuit realization and image encryption. Analog Integr Circuits Signal Process 98:85-99

2. Gaspard $P$ (1999) Microscopic chaos and chemical reactions. Phys A Stat Mech Appl 263:315-328

3. Nana B, Woafo $P(2015)$ Chaotic masking of communication in an emitter-relay-receiver electronic setup. Nonlinear Dyn 82:899

4. Njitacke ZT, Kengne J (2018) Complex dynamics of a 4D Hopfield neural networks (HNNs) with a nonlinear synaptic weight: coexistence of multiple attractors and remerging Feigenbaum trees. Int J Electron Commun (AEÜ) 93:242-252

5. Kyriazis M (1991) Applications of chaos theory to the molecular biology of aging. Exp Gerontol 26:569-572

6. Ren GD, Wu G, Ma J et al (2015) Simulation of electric activity of neuron by setting up a reliable neuronal circuit driven by electric autapse. Acta Phys Sin 64:058702

7. Wu XY, Ma J, Yuan LH et al (2014) Simulating electric activities of neurons by using PSPICE. Nonlinear Dyn 75:113-126

8. Korkmaz N, Ozturk I, Kilic R (2016) The investigation of chemical coupling in a HR neuron model with reconfigurable implementations. Nonlinear Dyn 86:1841-1854

9. Ren GD, Zhou P, Ma J et al (2017) Dynamical response of electrical activities in digital neuron circuit driven by autapse. Int J Bifurc Chaos 27:1750287

10. Lorenz EN (1963) Deterministic non-periodic flows. J Atmos Sci 20:130-141

11. Rössler OE (1976) An equation for continuous chaos. Phys Lett A 57:397-398

12. Pham VT, Ouannas A, Volos C, Kapitaniak T (2018) A simple fractional-order chaotic system without equilibrium and its synchronization. Int J Electron Commun (AEÜ) 86:6

13. Kengne J, Njitacke ZT, Fotsin HB (2015) Dynamical analysis of a simple autonomous jerk system with multiple attractors. Nonlinear Dyn. https://doi.org/10.1007/s11071-015-2364-y

14. Chlouverakis KE, Sprott JC (2006) Chaotic hyperjerk system. Chaos Solitons Fractals 28:739-747

15. Pham VT, Volos C, Jafari S, Wang X, Kapitaniak T (2016) A simple chaotic circuit with a light-emitting diode. Optoelectron Adv Mater Rapid Commun 10:640-646 
16. El-Nabulsi RA (2018) Time-nonlocal kinetic equations, jerk and hyperjerk in plasmas and solar physics. Adv Space Res 61:2914-2931

17. Barboza R (2007) Dynamics of a hyperchaotic Lorenz system. Int J Bifurc Chaos 17:4285-4294

18. Pham VT, Volos C, Kingni T, Kapitaniak T, Jafari S (2018) Bistable hidden attractors in a novel chaotic system with hyperbolic sine equilibrium. Circuits Syst Signal Process 37:1028-1043

19. Jafari S, Ahmadi A, Panahi S, Rajagopal K (2018) Extreme multistability: when imperfection changes quality. Chaos Solitons Fractals 108:182-186

20. Bowong S (2005) A new adaptive chaos synchronization principle for a class of chaotic systems. Int J Nonlinear Sci 6:399-408

21. Sprott JC, Jafari S, Jalil AM, Kapitaniak T (2017) Megastability: coexistence of a countable infinity of nested attractors in a periodically-forced oscillator with spatially-periodic damping. Eur Phys J Spec Top 226:1979-1985

22. Ma J, Fuqiang W, Ahmed A, Jun T (2018) Crack synchronization of chaotic circuits under field coupling. Nonlinear Dyn. https:// doi.org/10.1007/s11071-018-4307-x

23. Louodop P, Kountchou M, Fotsin H, Bowong S (2014) Practical finite-time synchronization of jerk systems: theory and experiment. Nonlinear Dyn 78:597-607

24. Megam NEB, Fotsin HB, Louodop FP, Kamdoum VT, Cerdeira $\mathrm{AH}$ (2016) Bifurcations and multistability in the extended Hindmarsh-Rose neuronal oscillator. Chaos Solitons Fractals 85:151-163

25. Li C, Chen G (2003) A note on hopf bifurcation in chen's system. Int J Bifurc chaos 13:1609-1615

26. Yan XP, Li WT (2006) Hopf bifurcation and global periodic solutions in a delayed predator-prey system. Appl Math Comput 177:427-445

27. Yu Y, Zhang S (2003) Hopf bifurcation in the Lü system. Chaos Solitons Fractals 17:901

28. Mello LF, Coelho SF (2009) Degenerate hopf bifurcations in the Lü system. Phys Lett A 373:1116-1120

29. Zhou X, Wu Y, Li Y, Wei Z (2008) Hopf bifurcation analysis of the Liu system. Chaos Solitons Fractals 36:1385-1391

30. Wei Z, Moroz I, Wang Z, Sprott JC, Kapitaniak T (2016) Dynamics at infinity degenerate Hopf and Zero-Hopf bifurcation for Kingni-Jafari system with hidden attractors. Int J Bifurc Chaos. https://doi.org/10.1142/S021812741650125X

31. Sprott JC (1997) Some simple chaotic jerk functions. Am J Phys 65:537-543

32. Sprott JC (1997) Simplest dissipative chaotic flow. Phys Lett $A$ 228:271-274

33. Sprott JC (2000) Simple chaotic systems and circuits. Am J Phys 68:758-763

34. Sprott JC, Linz SJ (2000) Algebraically simple chaotic flows. Int J Chaos Theory Appl 5:3-22

35. Von Baeyer HC (1998) All shook up: the jerk, an old-fashioned tools of physics, find new applications in the theory chaos. Sciences 38:12-14

36. Linz SJ (2000) No-chaos criteria for certain jerky dynamics. Phys Lett A 275:204-210

37. Alombah NH, Fotsin H, Megam EBN, Nguazon T (2016) Dynamics, analysis and implementation of a multiscroll memristorbased chaotic circuit. Int J of Bifurc Chaos 26:1650128

38. Megam EBN, Fotsin HB, Louodop F (2014) Implementing a memristive Van der Pol oscillator coupled to a linear oscillator: synchronization and application to secure communication. Phys Scr 89:035201

39. Woafo P, Kraenkel RA (2002) Synchronization: stability and duration time. Phys Rev E 65:362251-362256

40. Wiggins $S$ (1990) Introduction to applied nonlinear dynamical systems and chaos. Springer, New York

41. Kuznetsov YA (1998) Elements of applied bifurcation theory. Springer, New York
42. Hassard B, Kazarinoff N, Wan Y (1982) Theory and application of Hopf bifurcation. Cambridge University Press, Cambridge

43. Guckenheimer J, Holmes P (1983) Nonlinear oscillations, dynamical systems and bifurcation of vector field. Springer, New York

44. Qigui Y, Meili B (2016) A new 5D hyperchaotic system based on modified generalized Lorenz system. Nonlinear Dyn 88:189-221

45. Wolf A, Swift JB, Swinney HL, Wastano JA (1985) Determining Lyapunov exponents from time series. Phys D 16:285-317

46. Tamasevicius A, Namajunas A, Cenys A (1996) Simple 4D chaotic oscillator. Electron Lett 32:11

47. Kengne J, Jafari S, Njitacke ZT, Yousefi AK, Cheukem A (2017) Dynamic analysis and electronic circuit implementation of a novel 3D autonomous system without linear terms. Commun Nonlinear Sci Numer Simul. https://doi.org/10.1016/j.cnsns .2017.04.017

48. Njitacke ZT, Kengne J, Fotsin HB (2018) A plethora of behaviors in a memristor based Hopfield neural networks (HNNs). Int J Dyn Control. https://doi.org/10.1007/s40435-018-0435-x

49. Pham VT, Volos C, Kingni ST, Jafari S, Kapitaniak T (2017) Coexistence of hidden chaotic attractors in a novel no-equilibrium system. Nonlinear Dyn 87:2001

50. Bayani A, Rajagopal K, Jalil AK, Jafari S, Leutcho GD, Kengne J (2019) Dynamical analysis of a new multistable chaotic system with hidden attractor: antimonotonicity, coexisting multiple attractors, and offset boosting. Phys Lett A 13:1450-1456

51. Li C, Sprott JC, Akgul A, Lu HHC, Zhao Y (2107) A new chaotic oscillator with free control. Chaos 27:083101

52. Leutcho GD, Kengne J, Kengne R (2018) Remerging Feigenbaum trees, and multiple coexisting bifurcations in a novel hybrid diode-based hyperjerk circuit with offset boosting. Int J Dyn Control. https://doi.org/10.1007/s40435-018-0438-7

53. Dawson SP, Grebogi C, York JA, IKan I, Koçak K (1992) Antimonotonicity: inevitable reversals of period-doubling cascades. Phys Lett A 162:249-254

54. Kocarev L, Halle K, Eckert K, Chua L (1993) Experimental observation of antimonotonicity in Chua's circuit. Int J Bifurc Chaos 3:1051-1055

55. Parlitz U, Lauterborn W (1987) Period-doubling cascades and devil's staircases of the driven van der Pol oscillator. Phys Rev A 36:1428

56. Bier M, Bountis TC (1984) Remerging Feigenbaum trees in dynamical systems. Phys Lett A 104:239-244

57. Linz SJ (2008) On hyperjerk systems. Chaos Solitons Fractals $37: 741-747$

58. Rajagopal K, Jafari S, Pham VT, Wei Z, Premraj D, Thamilmaran K, Karthikegan A (2019) Antimonotonicity, bifurcation and multistability in the Vallis model for El Niño. Int J Bifurc Chaos 29:1950032

59. Kingni ST, Jafari S, Simo H, Woafo P (2014) Three dimensional chaotic autonomous system with only one stable equilibrium: analysis, circuit design, parameter estimation, control, synchronization and its fractional-order form. Eur Phys J Plus 129:76

60. Kingni ST, Keuninckx L, Woafo P et al (2013) Dissipative chaos, Shilnikov chaos and bursting oscillations in a three-dimensional autonomous system: theory and electronic implementation. Nonlinear Dyn 73:1111-1123

61. Kengne R, Tchitnga R, Mabekou S et al (2018) On the relay coupling of three fractional-order oscillators with time-delay consideration: global and cluster synchronizations. Chaos Solitons Fractals 111:6-17

62. Kengne J, Tabekoueng ZN, Tamba VK, Negou AN (2015) Periodicity, chaos, and multiple attractors in a memristor-based Shinriki's circuit. Chaos 25:103126-1-103126-10

Publisher's Note Springer Nature remains neutral with regard to jurisdictional claims in published maps and institutional affiliations. 